\title{
OPTIMIZATION AND EXPERIMENTAL TESTS OF A CENTRIFUGAL TURBINE FOR AN OWC DEVICE EQUIPPED WITH A TWIN TURBINES CONFIGURATION
}

\author{
Laudino Rodrígueza, Bruno Pereiras ${ }^{\mathrm{b}, *}$, Jesús Fernández-Oro ${ }^{\mathrm{b}}$ and Francisco Castroc \\ a (1) Integrated center of F.P. Maintenance and Production Services (C.I.F.P.M.S.P.) \\ Ciudad Tecnológica e Industrial Valnalón, Hornos Altos S/N, 33930, La Felguera, Spain \\ laudelinrg@gmail.com
}

${ }^{b}$ Department of Energy, University of Oviedo

Energy Building (EDZE), Campus de Viesques s/n, 33271, Gijón, Spain

*pereirasbruno@uniovi.es; jesusfo@uniovi.es

${ }^{c}$ Department of Energetic and Fluidmechanics Engineering. University of Valladolid

Paseo del Cauce 59, 47011, Valladolid, Spain

castro@eii.uva.es

\section{ABSTRACT}

The optimization of OWC devices has deserved much attention in the last few years. However, despite of this intense research activity, the most suitable turbine for OWC applications is still under debate. Recently, the twinturbine configuration, where two unidirectional turbines are employed simultaneously, has emerged as a promising design. Although axial turbines are typically employed for those systems, the present paper demonstrates that the use of radial turbines can be also an interesting option.

Experimental data on radial turbine performance for OWC applications is quite scarce in the literature. Since experimental results are essential to validate both numerical studies and theoretical estimations, more experimental testing is necessary for these new developments. In this work a radial geometry, specifically designed to be used in a twin-turbine configuration, has been manufactured at a reasonable cost for a lab-scale facility taking advantage of 3D printing technology.

Encouraging preliminary results were obtained in an aerodynamic database of the turbine. In particular, the totalto-static efficiency under stationary conditions (i.e. at constant flow coefficients) reached remarkable high values. Hence, the performance curve of the turbine under such stationary conditions has been used to make an assessment of its non-stationary performance in order to compare this new radial turbine with respect to axial types available in the literature. The results revealed that radial turbines are clearly competitive against to axial ones when introduced in a twin-turbine configuration for OWC power plants. 


\begin{tabular}{|c|c|c|c|c|c|}
\hline \multicolumn{6}{|c|}{ NOMENCLATURE } \\
\hline$A_{r}=\pi b D_{m}$ & Reference area & $\mathrm{m}^{2}$ & $u_{R}=D_{m} \cdot \omega / 2$ & Reference tangential velocity & $\mathrm{m} / \mathrm{s}$ \\
\hline b: & Blade Span & $\mathrm{m}$ & $V_{A B}:$ & Tachogenerator terminal voltage & $\mathrm{V}$ \\
\hline $\mathrm{C}_{\mathrm{A}}:$ & Input coefficient & & $\mathrm{V}_{\mathrm{R}}=\mathrm{Q} /\left(\pi \cdot \mathrm{b} \cdot \mathrm{D}_{\mathrm{m}}\right)$ & Reference radial velocity & $\mathrm{m} / \mathrm{s}$ \\
\hline $\mathrm{C}_{\mathrm{T}}:$ & Torque coefficient & & $W_{A B}:$ & Power in terminals of tachogenerator & W \\
\hline DIN: & Inlet diameter of turbine & $\mathrm{m}$ & $\mathrm{W}_{\text {cu: }}:$ & Joule losses & W \\
\hline$D_{m}:$ & Mean diameter of turbine & $\mathrm{m}$ & We: & Electric power & W \\
\hline FEM: & Electromotive force & $\mathrm{V}$ & W $_{\mathrm{FE}}:$ & Iron losses & W \\
\hline $\mathrm{I}:$ & Tachogenerator Intensity & A & WMEC: & Mechanical losses & W \\
\hline lb: & Chord blade & $\mathrm{m}$ & $\mathrm{W}_{\mathrm{IN}}=\Delta \mathrm{Pt}-\mathrm{e} \mathrm{VR}_{\mathrm{R}} \mathrm{A}_{\mathrm{r}}$ & Input power & W \\
\hline $\mathrm{N}:$ & Tachometer rotation speed & rpm & Wout: & Outlet power & W \\
\hline P: & Barometric pressure & $\mathrm{hPa}$ & Wr: & Resistant power & W \\
\hline$P_{e}:$ & Static pressure & $\mathrm{Pa}$ & Z: & Blades number & \\
\hline Powc: & Pressure in OWC chamber & $\mathrm{Pa}$ & $\beta_{1}, \beta_{2}:$ & Inner and outer blade angles & \\
\hline Q: & Flow rate & $\mathrm{m}^{3} / \mathrm{s}$ & $\Delta$ Pt-e: & Total to static pressure drop & $\mathrm{Pa}$ \\
\hline$Q_{D}:$ & Direct flow rate & $\mathrm{m}^{3} / \mathrm{s}$ & $\Delta \mathrm{P}_{\mathrm{e}}:$ & Static pressure drop & $\mathrm{Pa}$ \\
\hline QR: & Reverse flow rate & $\mathrm{m}^{3} / \mathrm{s}$ & $\bar{\eta}_{\text {input }}:$ & Input efficiency & \\
\hline QT: & Total flow OWC chamber & $\mathrm{m}^{3} / \mathrm{s}$ & $\bar{\eta}_{\text {system }}:$ & Efficiency of the whole system & \\
\hline $\mathrm{R}_{1}, \mathrm{R}_{2}$ : & Blade curvature & & $\bar{\eta}_{\text {turbine }}$ & Net efficiency of the twin turbines & \\
\hline Rinner, Router & Inner and outer rotor dimensions & & $\eta_{v}$ & Volumetric efficiency & \\
\hline $\mathrm{Ri}:$ & Tachogenerator internal resistance & $\Omega$ & $\rho:$ & Air density & $\mathrm{Kg} / \mathrm{m}^{3}$ \\
\hline $\mathrm{T}:$ & Wave period & $\mathrm{s}$ & $\sigma:$ & Rotor solidity & \\
\hline $\mathrm{T}_{0}:$ & Total torque & $\mathrm{N} \cdot \mathrm{m}$ & $\phi:$ & Flow coefficient & \\
\hline $\mathrm{T}_{\mathrm{OD}}:$ & torque in direct mode & $N \cdot m$ & $\Phi_{\mathrm{T}}:$ & Flow coefficient of twin system & \\
\hline $\mathrm{T}_{\mathrm{OR}}:$ & torque in reverse mode & $\mathrm{N} \cdot \mathrm{m}$ & $\omega:$ & Angular velocity & $\mathrm{rad} / \mathrm{s}$ \\
\hline
\end{tabular}

\section{1. Introduction}

34 Ocean's energy shows high potential for mid/long-future to make an important contribution to low-carbon

35 electricity generation. Due to the technological barriers, only small facilities, mainly for research purposes,

36 are operative at the present time, so a large amount of energy is still available for harnessing if those barriers 
are crossed. On the other hand, the marine environment is really challenging and very aggressive to the assembly and maintenance of ocean energy converters. In spite of this, different technologies to harvest these resources have been analysed in the last years (Falcão, 2010; IPCC, 2012), with the wave energy emerging as the most interesting option due to its high energy density (Drew et al., 2009), location possibilities (onshore, nearshore, offshore) and relative low environmental impact (Iglesias and Carballo, 2014; Palha et al., 2010).

A wide number of patents for wave energy converters (WEC) has been registered in the last decades, proposing different alternatives for the exploitation of wave energy. (Drew et al., 2009; Falcão, 2010; López et al., 2013). In particular, the OWC device is probably the most widespread selection due to its installation possibilities and the less exposure to critical sea conditions (Falcão and Henriques, 2016). The basic principle of an OWC device is the conversion of wave energy into pneumatic energy in a submerged chamber opened at the bottom to the incoming waves (Figure 1). The waves produce an towards the chamber. These processes, called exhalation and inhalation, take place sequentially according to the wave motion, generating a pulsating flow which can be used to drive an air turbine and thus generate electricity.

The main feature of OWC devices is the non-steady airflow conditions: the change of the airflow direction within every wave cycle as well as the oscillating variation of its magnitude. In particular, the wide range of instantaneous operating conditions is the key for the design of efficient turbines used as Power Take Off (Raghunathan, 1995; Wells, 1980) and impulse turbines (Setoguchi and Takao, 2006), both broadly referenced in the literature concerning OWC devices: Well turbines with or without guide vanes and/or 
Henriques, 2016; Setoguchi and Takao, 2006). Nevertheless, the poor efficiencies of bidirectional turbines have led to the development of a relatively new concept: the twin turbine configuration (Figure 1), where two unidirectional turbines are assembled to the same rotating axis. Since the turbines are installed to provide an opposite performance, one of the turbines must produce energy when the air is exhaled, while the other has to drive power when the air is inhaled. Note that due to the absence of non-return valves, a special design is required for the turbines to prevent the reverse flow. At present time, numerical and experimental studies of twin-turbine systems have been carried out on axial turbines only (Jayashankar et al., 2009; Mala et al., 2011; Pereiras et al., 2014; Takao et al., 2011; Takao and Setoguchi, 2012). However, in spite of being known that radial turbines for OWC work under larger pressure differences than axial ones (Pereiras et al., 2011; Setoguchi and Takao, 2006), what could be a real advantage working as backflow preventers , there are hardly any studies (Rodríguez et al., 2018) on the radial-flow turbine for twin systems.

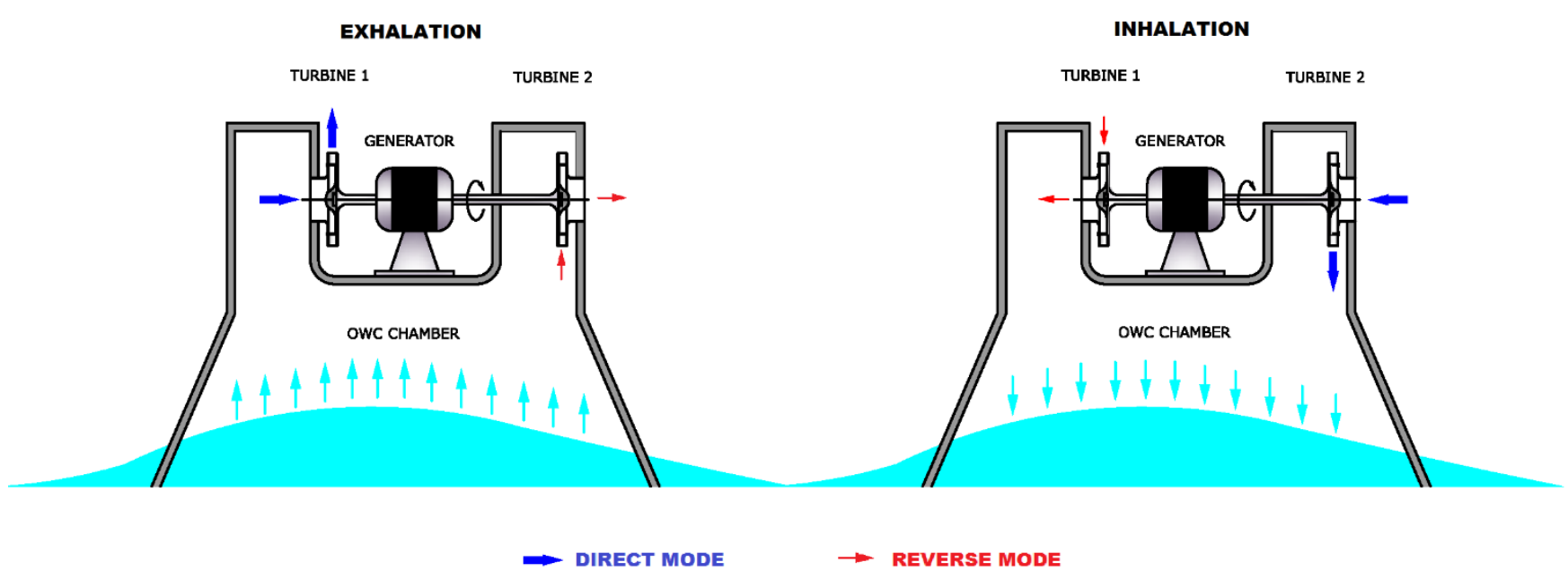

78

Figure 1 Twin turbines system layout

A preliminary evaluation of the performance of radial turbines for twin systems has been recently published by the authors (Rodríguez et al., 2018) . The restrictions observed during the analysis of that initial design has led to the development and construction of a brand-new model which increases the turbine efficiency and strengthens its performance as backflow preventer. In this paper, the new geometry, the manufacturing conception, the experimental rig and the testing procedure are presented. Additionally, the results from a test campaign in steady conditions are shown and a non-steady analysis based on such steady results is finally made in order to compare this new turbine to those available within the bibliography. 


\section{Dimensionless coefficients}

In the literature the performance of the turbine in steady conditions is assessed using the following nondimensional coefficients:

$$
\phi=\frac{v_{R}}{u_{R}} \quad C_{T}=\frac{4 \cdot T_{o}}{\rho \cdot\left(v_{R}^{2}+u_{R}^{2}\right) \cdot \pi \cdot b \cdot D_{m}^{2}} \quad C_{A}=\frac{2 \cdot W_{I N}}{\rho \cdot\left(v_{R}^{2}+u_{R}^{2}\right) \cdot \pi \cdot b \cdot D_{m} \cdot v_{R}} \quad \eta=\frac{C_{T}}{C_{A} \cdot \phi}
$$

Being $\phi$ the flow coefficient, $C_{T}$, the torque coefficient, $C_{A}$ the so-called input coefficient and $\eta$ the total-tostatic efficiency. The detailed definitions of the variables introduced in these coefficients are given in the nomenclature section.

On the other hand, the wave energy converter behaves non-steadily with typical time scales in the order of the waves period, so a temporal description of its relevant parameters is also needed. Fortunately, the time scales within the turbine stages (i.e., the blade passing frequencies) are two or three orders of magnitude lower than the global fluctuations in the OWC system (the incoming waves at the boundaries), so it can be perfectly assumed that the turbine works under quasi-steady boundary conditions in regard to the wave time scales (Inoue et al., 1986; Takao et al., 2011). This means that the steady characterization of the turbine can be used as input data to perform a further non-steady analysis of the whole system, where the input variable is the manometric static pressure inside the chamber defined according to a sinusoidal wave motion as:

$$
P_{O W C}=P_{\max } \cdot \sin \left(\frac{2 \pi t}{T}\right)
$$

Where $P_{\max }$ is the maximum pressure at the OWC within a wave cycle, $T$ is the period of the wave and $t$ is the current time.

During operation, both turbines of the twin system are exposed to the same pressure difference. Their performance is switched from direct to reverse mode and vice versa according to the sign of the pressure difference. Hence, the total outgoing flow rate transferred from the chamber is the combination of both direct $\left(Q_{D}\right)$ and reverse $\left(Q_{R}\right)$ mode flowrates:

$$
Q_{T}=Q_{D}+Q_{R}
$$

So, the volumetric efficiency of the OWC can be determined as the ratio between those direct and the total flow rates, according to:

$$
\eta_{V}=\frac{Q_{D}}{Q_{T}}=\frac{Q_{D}}{Q_{D}+Q_{R}}
$$


118 However, the turbine efficiency is also relevant, so it must be evaluated in combination with the efficiency 119 of the twin system. This can be assessed according to the following expression:

$$
\bar{\eta}_{\text {system }}=\frac{\frac{1}{T} \int_{0}^{T} \omega \cdot T_{o} \cdot d t}{\frac{1}{T} \int_{0}^{T} \Delta P_{t-e} \cdot Q_{T} \cdot d t}=\underbrace{\frac{\frac{1}{T} \int_{0}^{T} \Delta P_{t-e} \cdot Q_{D} \cdot d t}{\frac{1}{T} \int_{0}^{T} \Delta P_{t-e} \cdot Q_{T} \cdot d t}}_{\bar{\eta}_{\text {input }}} \cdot \underbrace{\frac{1}{T} \int_{0}^{T} \omega \cdot\left(T_{o D}-T_{o R}\right) \cdot d t}_{\bar{\eta}_{\text {turbine }}}
$$

Or more compactly:

$$
\bar{\eta}_{\text {system }}=\bar{\eta}_{\text {input }} \cdot \bar{\eta}_{\text {turbine }}
$$

Note that the so-called input efficiency, $\bar{\eta}_{\text {input }}$ is related to the volumetric efficiency $\left(\eta_{V}\right)$ of the OWC chamber, but in terms of power instead of flow rates only; and that the turbine efficiency, $\bar{\eta}_{\text {turbinne }}$ is the net efficiency of the twin turbines, considering the resistant torque $\left(T_{O R}\right)$ produced in the reverse mode. Alternatively, the system flow coefficient is also defined as the ratio between the mean flow velocity of both turbines and the reference tangential velocity in the turbine mean diameter of the turbine:

$$
\Phi_{T}=\frac{Q_{T}}{\pi \cdot D_{m} \cdot b \cdot u_{R}}
$$

Where $Q_{T}$ is the total flow rate, $D_{m}$ is the mean diameter of turbine, $b$ is the blade span and $u_{R}$ is the reference tangential velocity at midspan.

\section{Geometry optimization}

To enhance the performance of a radial turbine for the twin-system configuration, a geometry optimization has been firstly completed, taking advantage of a previous CFD analysis (Rodríguez et al., 2018). The model of the turbine is composed of three main parts: elbow, rotor and diffuser, was initially designed to work with a centrifugal flow (or "direct mode" operation, from now on). Figure 2 shows a 3D view of the model (a single passage with circumferential periodicity) and the corresponding boundary conditions (more details can be found in (Rodríguez et al., 2018). 


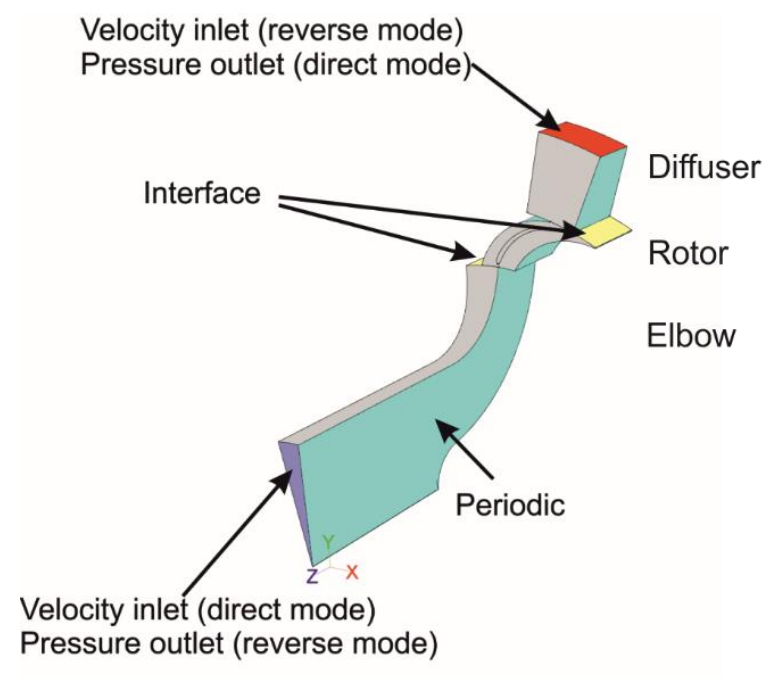

Figure 2. CFD model

When this turbine, installed in the twin configuration, is to be operated in the centripetal direction (or "reverse mode"), it is extremely important that the turbine could perform as an optimal backflow preventer to maximize the overall efficiency of the system. Thus, a design criterion can be declared as the larger the input coefficient in reverse mode, the better performance as backflow preventer.

In (Rodríguez et al., 2018) it was revealed that this kind of radial turbine shows several strong points, making it suitable for working in a twin turbines configuration. Nevertheless, some penalties were also observed after analyzing the results in the first numerical model, mainly related to a weak performance when working in reverse mode as a backflow preventer (1) and an excessive kinetic energy loss at the diffuser in direct mode (2).

Consequently, for this investigation, a re-design of the original geometry has been carried out to include some new modifications in the turbine and correct all those detrimental problems. In particular I.

a) To improve the performance as a backflow preventer in reverse mode: The suppression of three structural ribs, originally introduced for rigidity, in the connection of the shroud at the elbow (Figure 3), which were acting as guide vanes during the reverse mode, breaking the swirl generated rotor downstream, and thus reducing the (desirable) loss at the elbow. A complementary CFD model of the single elbow (with and without ribs) demonstrates the significant improvement of the unribbed elbow with higher losses helping to strengthen the performance as a backflow preventer (Figure 4), and enhance the twin system efficiency. Therefore, any guide vanes/ribs were suppressed in order to optimize the performance as a backflow preventer in the 
reverse mode. Overcoming this issue forced a change in the mechanical conception of the turbine (see Figure 8 later) with respect to that shown in (Rodríguez et al., 2018).
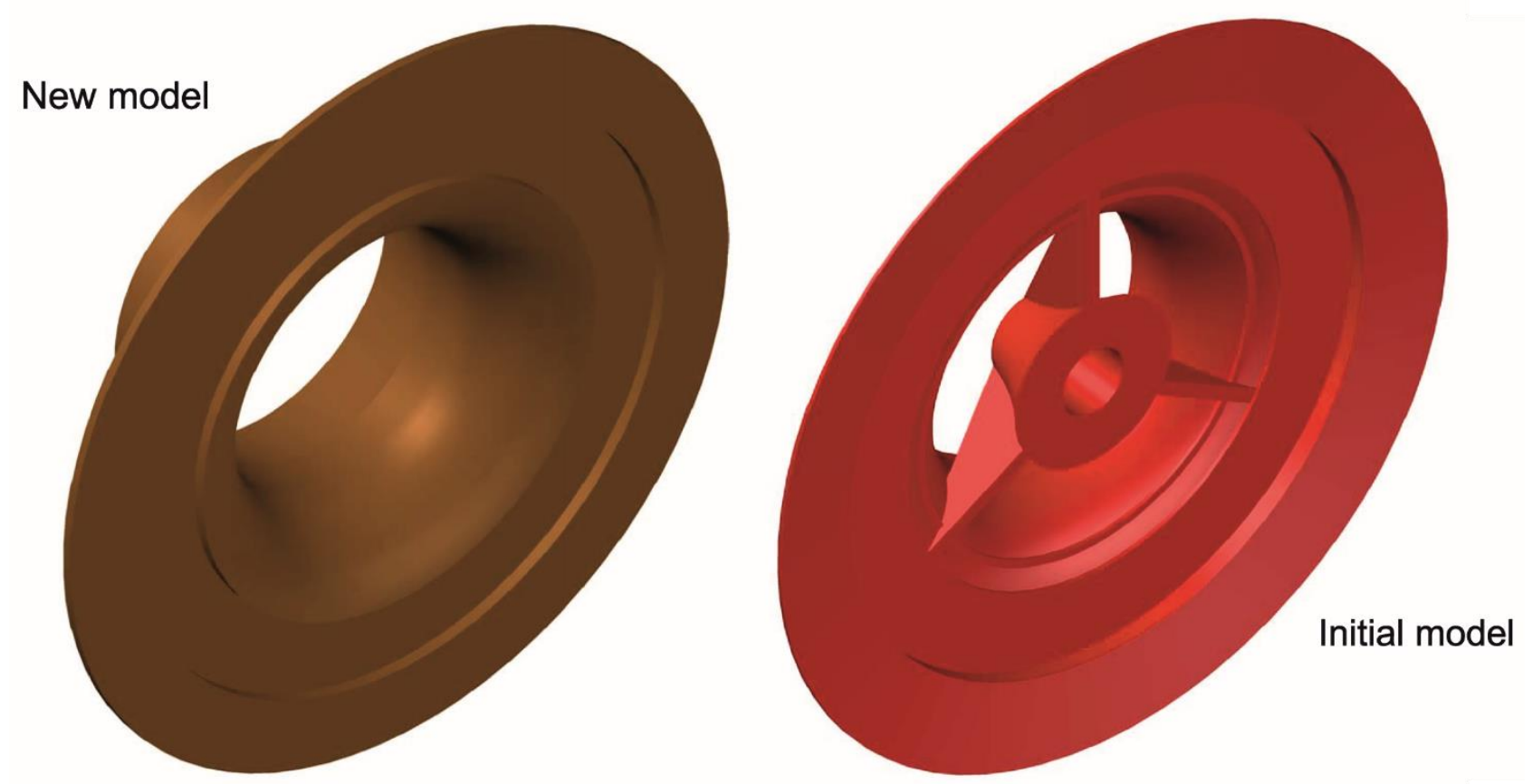

Figure 3. Connection of the shroud at the elbow.

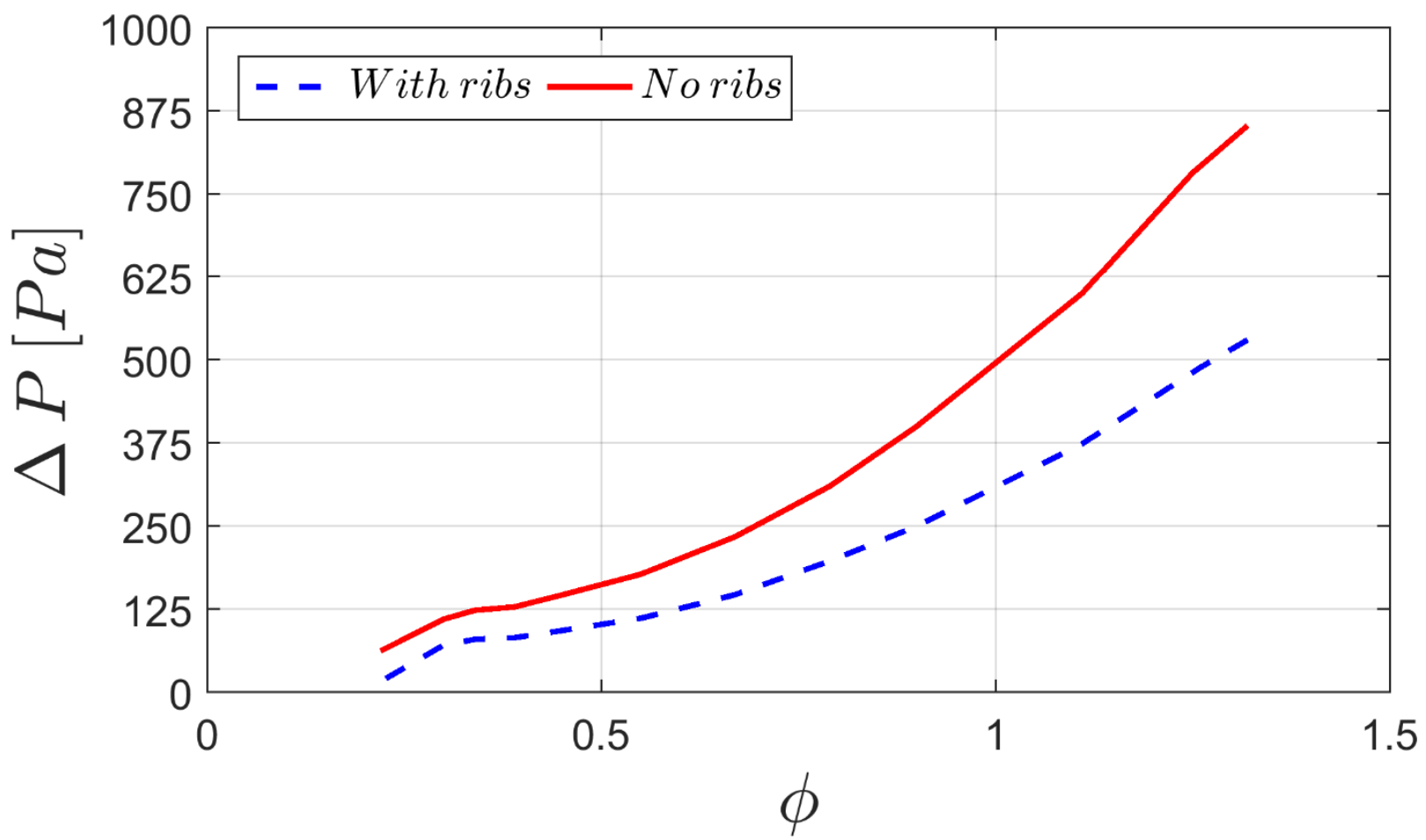

Figure 4. Effect of the structural ribs in the elbow loss during reverse mode. 
b) To reduce the kinetic energy loss at the outlet in direct mode

One of the most remarkable problem of the original geometry was the large difference between the total-to-total and the total-to-static efficiency when working in direct mode. After an initial reconsideration of the diffuser, the in-depth analysis of the numerical results redirected the attention towards the rotor outlet.

Therefore, in order to obtain a real reduction of the kinetic energy at the outlet, actions were taken on the rotor, focusing on the blade profile and the rotor solidity. In the original model the outer angle $\left(\beta_{2}\right.$, Figure 5$)$ of the blade was fixed to 5 degrees, resulting in very closely spaced blades, a very reduced outlet section and a really high velocity magnitude at the outlet. Several CFD simulations were then conducted for various blade profiles equipped with different $\beta_{2}$ while maintaining the inlet angle $\left(\beta_{1}\right)$ to 65 degrees, aiming to find the optimal $\beta_{2}$ for this application. Note that, since the kinetic energy was affecting the performance of the direct mode, simulations were carried out in the centrifugal direction only, using the rotor efficiency as the relevant parameter for the optimization.

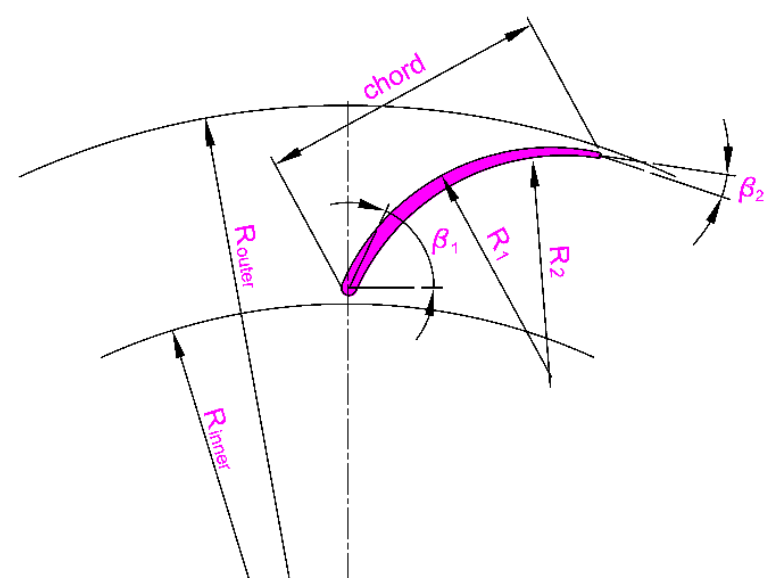

Figure 5. Blade profile.

The increment of the maximum efficiency obtained with the modified blade profile with respect to the original airfoil is plotted as a function of the blade external angle $\left(\beta_{2}\right)$ in Figure 6. A parabolic fitting was finally used to determine the optimum blade angle, corresponding to 11 deg for a $9 \%$ higher efficiency. 


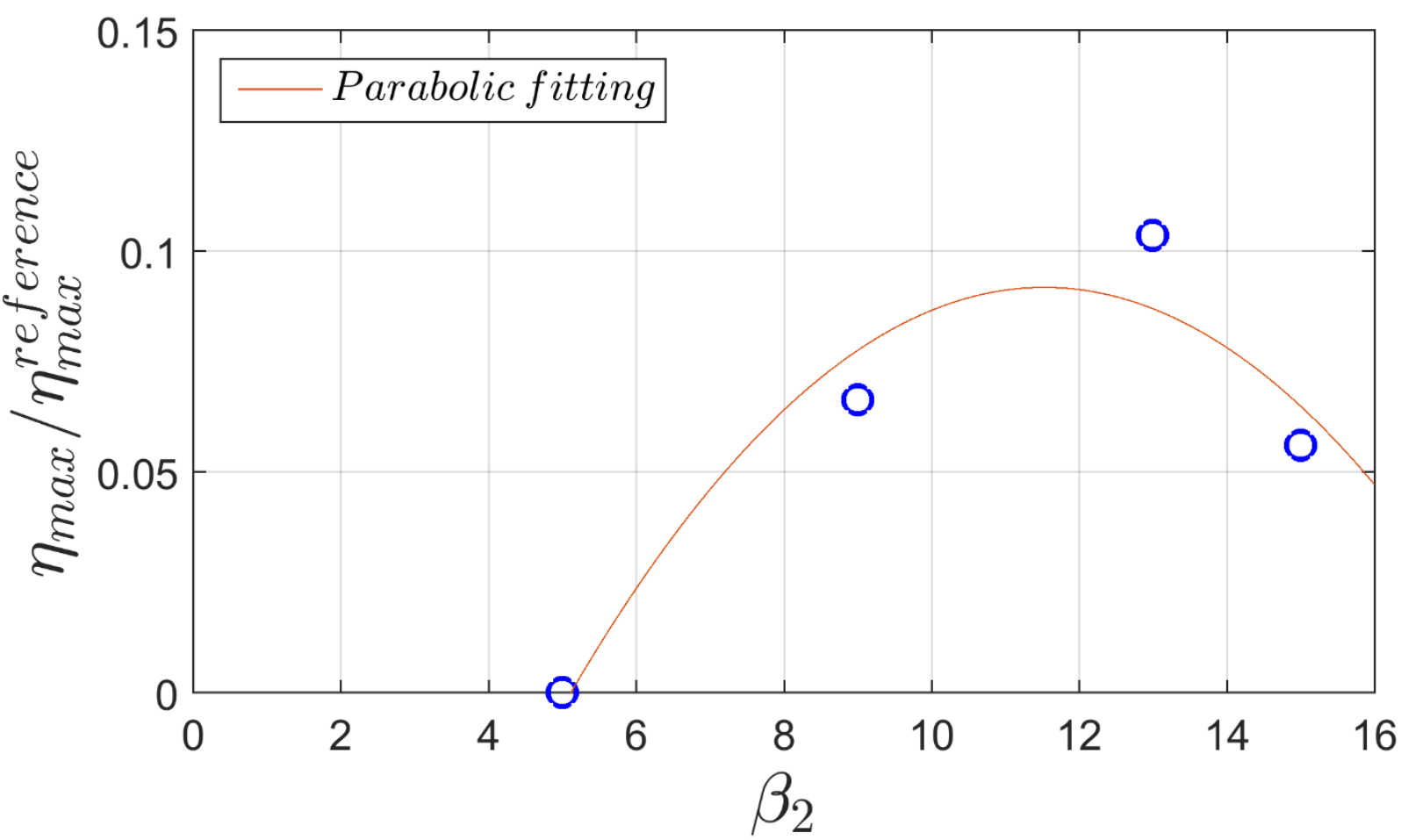

189

Figure 6. Increment of the maximum efficiency in direct mode with respect to the external blade angle $\beta_{2}$

Alternatively, it was also found that the rotor solidity plays a major role in the turbine performance. Furthermore, not only presents a relevant impact on the efficiency in the direct mode, but it is also crucial in the performance of the turbine as a backflow preventer during the reverse mode. To quantify that influence, several simulations were additionally carried out for different rotor solidities, 1.83, 2.20 and 2.75, which correspond to 20,24 and 30 blades respectively. The rotor solidity was defined as:

$$
\sigma=\frac{l_{B} \cdot Z}{\pi \cdot D_{m}}
$$

Where $I_{B}$ is the blade chord, $Z$ is the number of blades and $D_{m}$ is the rotor mean diameter.

The results, shown in Figure 7, reveal that the overall efficiency $\bar{\eta}_{\text {system }}$ is quite similar for the whole set of cases. Actually, since there are many effect involved, some comments are needed at this point. It has been confirmed that increasing the solidity improves the guidance of the flow and lead to gain efficiency in direct mode in spite of having larger velocities at the rotor outlet due to the finite thickness of the blades. In addition, the performance as a backflow preventer is also affected because larger solidities reduce the vorticity at the elbow in reverse mode reducing the $\bar{\eta}_{\text {input }}$. Both effects compensate each other to show no 


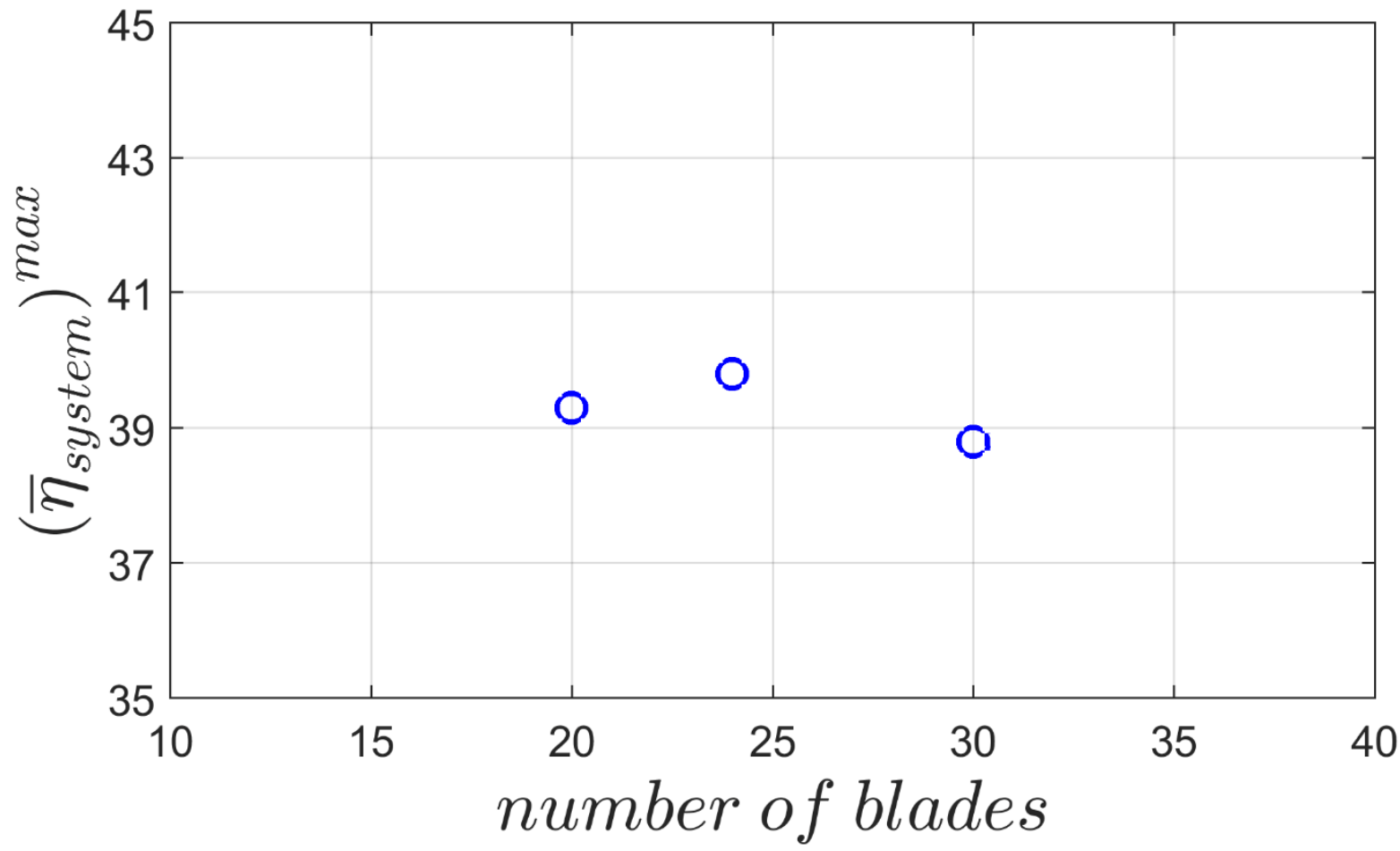

Figure 7. Influence of the rotor solidity on the twin system performance.

210 Considering these results, the intermediate solution was adopted for the new design, using 24 blades with 211 the following characteristics.

Table 1. Main dimensions [mm] of the new blade

\begin{tabular}{|l|l|}
\hline Chord & 43.2 \\
\hline$R_{1}=R_{2}$ & 28.8 \\
\hline$R_{\text {inner }}$ & 75 \\
\hline$R_{\text {outer }}$ & 100 \\
\hline$\beta_{1}$ & $65 \mathrm{deg}$ \\
\hline$\beta_{2}$ & $11 \mathrm{deg}$ \\
\hline
\end{tabular}




\section{4. Experimental tests}

215 The final concept of the manufactured model is shown in Figure 8, where the whole set of elements that 216 compose the model are exploded. The number of moving parts has been minimized to simplify operation 217 and reduce maintenance costs. Rotating parts (2, 3, 9 and 10) are mounted on the shaft (5) which is 218 supported by a ball bearing (7). The whole set of moving parts, including the diffuser as well, is fixed by one 219 single nut (8). The inlet connection (1), separated from the rest of the components, is assembled coaxially with the rest.
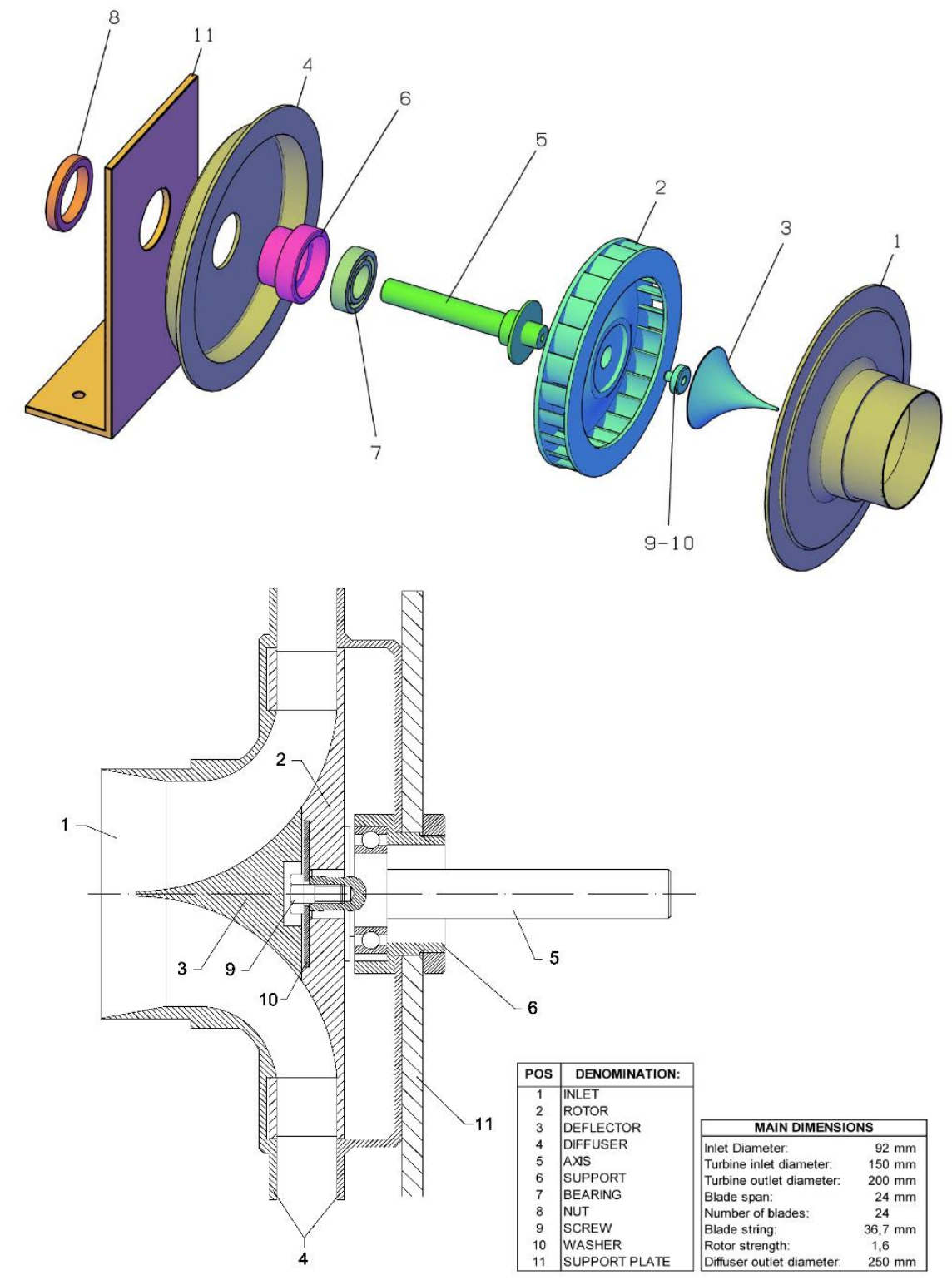

Figure 8. Conceptual model of turbine 
The geometrical dimensions of the turbine main components are referred at the bottom of Figure 8. Most of the non-commercial components $(1,2,3,4 a, 4 b)$ have been designed with the help of CAD software and built in a 3D printer model Witbox (manufacturer $\mathrm{BQ}$ ). Precisely, the turbine has been printed taking advantage of the whole printer capacity of $297 \times 210 \times 200 \mathrm{~mm}$ (length $\mathrm{x}$ width $\mathrm{x}$ height) and the maximum print quality: 50 microns. Under these conditions, the printer spent 57 hours to complete the whole assembly using PLA (biodegradable polylactic acid derived from corn starch, sugar cane, etc.) as the building material. The shaft, the bracket and the nuts have been manufactured in aluminum, using a conventional machinetool while the support plate (11) is made of steel. The bearing, the screw and the washer are standard commercial elements.

The upper Figure 9 shows the elements 1,2, 3 and 4 built with 3D printer, while the bottom Figure 9 shows the turbine assembly on the test bench.
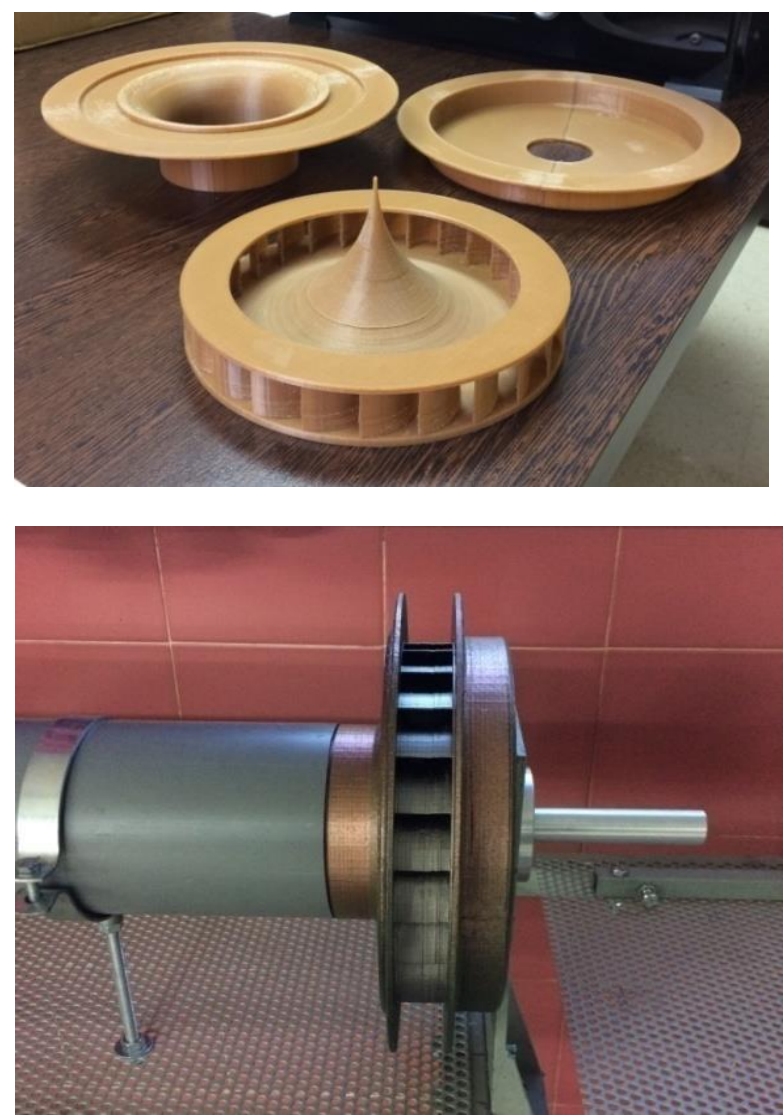

Figure 9. Top: components built with the 3D printer. Bottom: Turbine assembled.

A sketch of the setup is shown in Figure 10. The whole rig, mounted on a steel structure, is made of PVC tubes (3-6) with $103 \mathrm{~mm}$ in diameter. The air flow is supplied by a centrifugal fan (2) connected to a frequency inverter (15) in order to obtain variable flow rates. According to the manufacturer data the fan 
provides a maximum flow rate of $1250 \mathrm{~m}^{3} / \mathrm{h}$ (free exit) and maximum static pressure (at zero flow rate) of $1600 \mathrm{~Pa}$. The flow rate is measured with a Venturimeter (5) using a testo 435/9 (9) differential pressure sensor. To reduce turbulence levels and assure an inlet unidirectional velocity, a honeycomb is also installed between the centrifugal fan and the Venturimeter

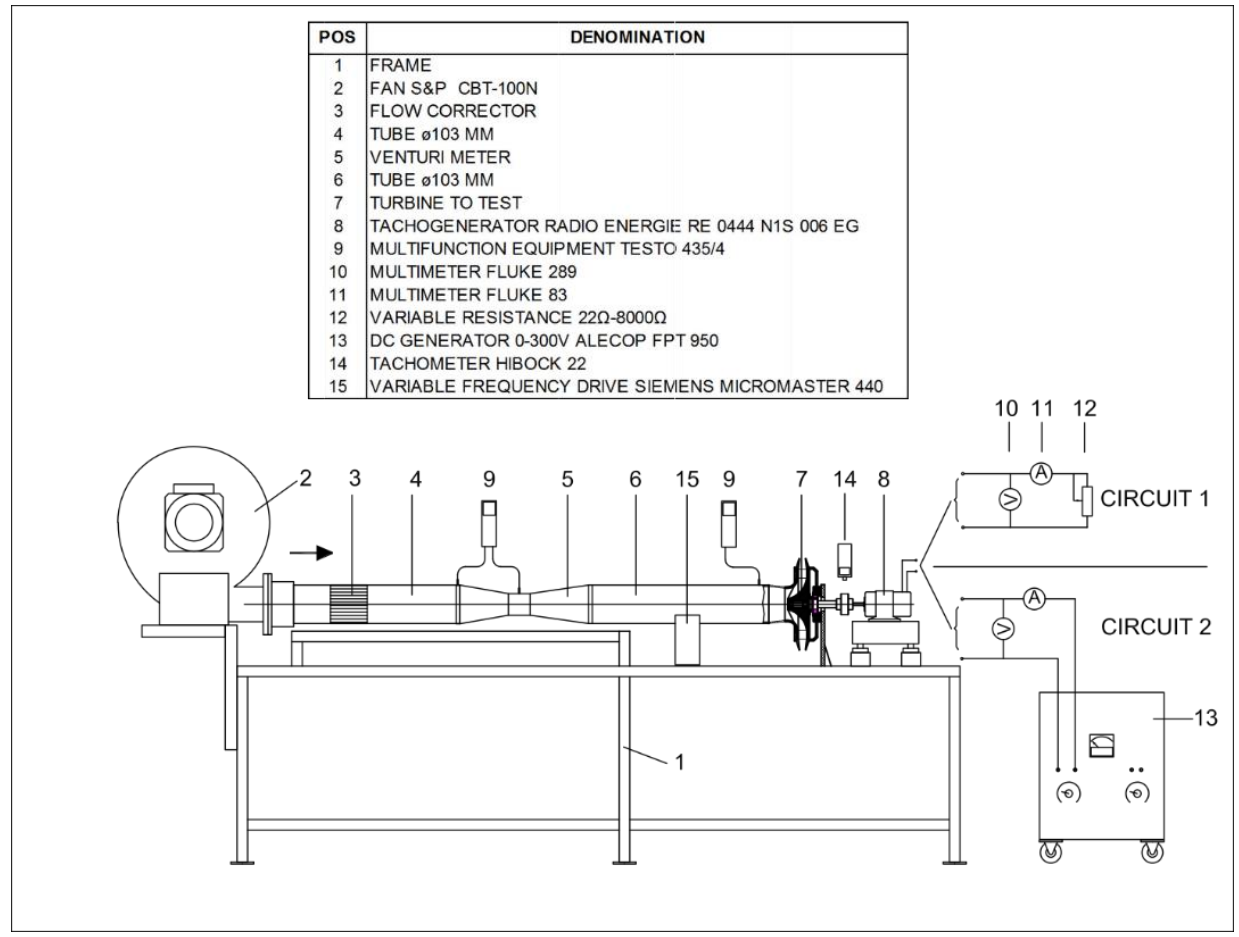

Figure 10. Experimental set up.

The experimental tests were carried out with a tachogenerator (8) running as a generator or a motor depending on the test to be made. To perform as a generator, the tachogenerator was connected to a grid of resistors using circuit no.1 (Figure 10) so the power generated by the turbine (7) could be dissipated for a variable range of loads (from 22 to $80 \mathrm{k} \Omega$ ). When the tachogenerator is required to run as a motor it is connected to a DC power supply (13) using circuit no 2 . In this case, the required rotational speed is obtained modifying the voltage of the DC power supply. Additional measuring devices are both Fluke 83 and 289 multimeters (10 and 11) that provide the measurements of voltage and current respectively. Besides, the torque and the rotating speed of the turbine were given by the tachogenerator (8) whose basic characteristics are shown in Table 2. Note that in the case of free rotation tests (i.e., the tachogenerator is disconnected), the rotating speed is measured with an optical tachometer (14), model Hibock 22. 
Table 2. Tachogenerator specifications

\begin{tabular}{ll|}
\hline Maker: & Predilec Radio Energie \\
Model: & RE 0444 N1S 006 EG \\
wmax: & $10000 \mathrm{rpm}$ \\
Vmax: & $600 \mathrm{~V}$ \\
V/w: & $0,06 \mathrm{~V} / \mathrm{rpm}$ \\
Ri: & $100 \Omega$ \\
Imax: & $0,180 \mathrm{~A}$ \\
Wmax: & $108 \mathrm{~W}$ \\
\hline
\end{tabular}

263 Finally, the atmospheric pressure, temperature and humidity are measured with a Weather station DAVIS 264 VANTAGE VUE 6351 and multifunction Testo $435 / 4$ in order to calculate the air density. Details of the accuracy of measurement equipment are shown in the Table 3.

Table 3. Accuracy of measurement equipment

\begin{tabular}{|c|c|c|c|}
\hline \multicolumn{4}{|c|}{ ACCURACY OF MEASUREMENT EQUIPMENT } \\
\hline Magnitude & Symbol & Measurement equipment & Minimum accuracy \\
\hline Barometric pressure & $P$ & Station DAVIS VANTAGE VUE 6351 & $\mathrm{hPa}$ \\
\hline Room temperature & $\mathrm{t}$ & Testo $435 / 4$ & $\pm 0,3$ \\
\hline Relative humidity & $\mathrm{Hr}$ & Testo $435 / 4$ & \pm 2 \\
\hline Static pressure inlet turbine & $\mathrm{Pe}$ & Testo $435 / 4$ & \pm 2 \\
\hline Differential pressure in Venturi & $\Delta \mathrm{Pe}$ & Testo $435 / 4$ & $\mathrm{~Pa}$ \\
\hline Angular velocity & $\omega$ & Tachometer Hibok 22 & $\pm 0,15$ \\
\hline Voltage DC & $\mathrm{V}$ & Multimeter Fluke 83 & $\pm(0,1 \%+0,1)$ \\
\hline Intensity DC & I & Multimeter Fluke 289 & $\pm(0,15 \%+0,02) \quad \mathrm{mA}$ \\
\hline Resistance DC & $\mathrm{R}$ & Multimeter Fluke 83 & $\pm(0,4 \%+0,1)$ \\
\hline Tachogenerator terminal voltage & $\mathrm{V}_{\text {TD }}$ & Tachogenerator RE 0444 N1S 006 EG & \pm 1 \\
\hline
\end{tabular}

The impact of the uncertainty of those measuring devices towards the accuracy of the complete measuring chain has been estimated using Klein's theory on uncertainty transmission (Marigorta and Tajadura, 1994). 
Hence, the turbine performance is complemented with the expected uncertainties in every working point of

272 the steady experimental tests, as it will be shown later in Figure 13 and Figure 14.

274 5. Experimental tests

275 The performance curves of the turbine under steady conditions for both direct and reverse modes have been obtained experimentally. For that purpose, it was necessary to conduct different types of tests in order to address the contribution of friction losses. In particular, the following tests are described in this section.

a) A tachogenerator loss test (in order to determine mechanical/electrical loss of the tachogenerator)

b) A turbine + tachogenerator loss test (in order to determine mechanical loss of the turbine)

c) Both turbine tests in direct mode (centrifugal) and reverse mode (centripetal) to determine the turbine performance

Table 4. List of measured variables.

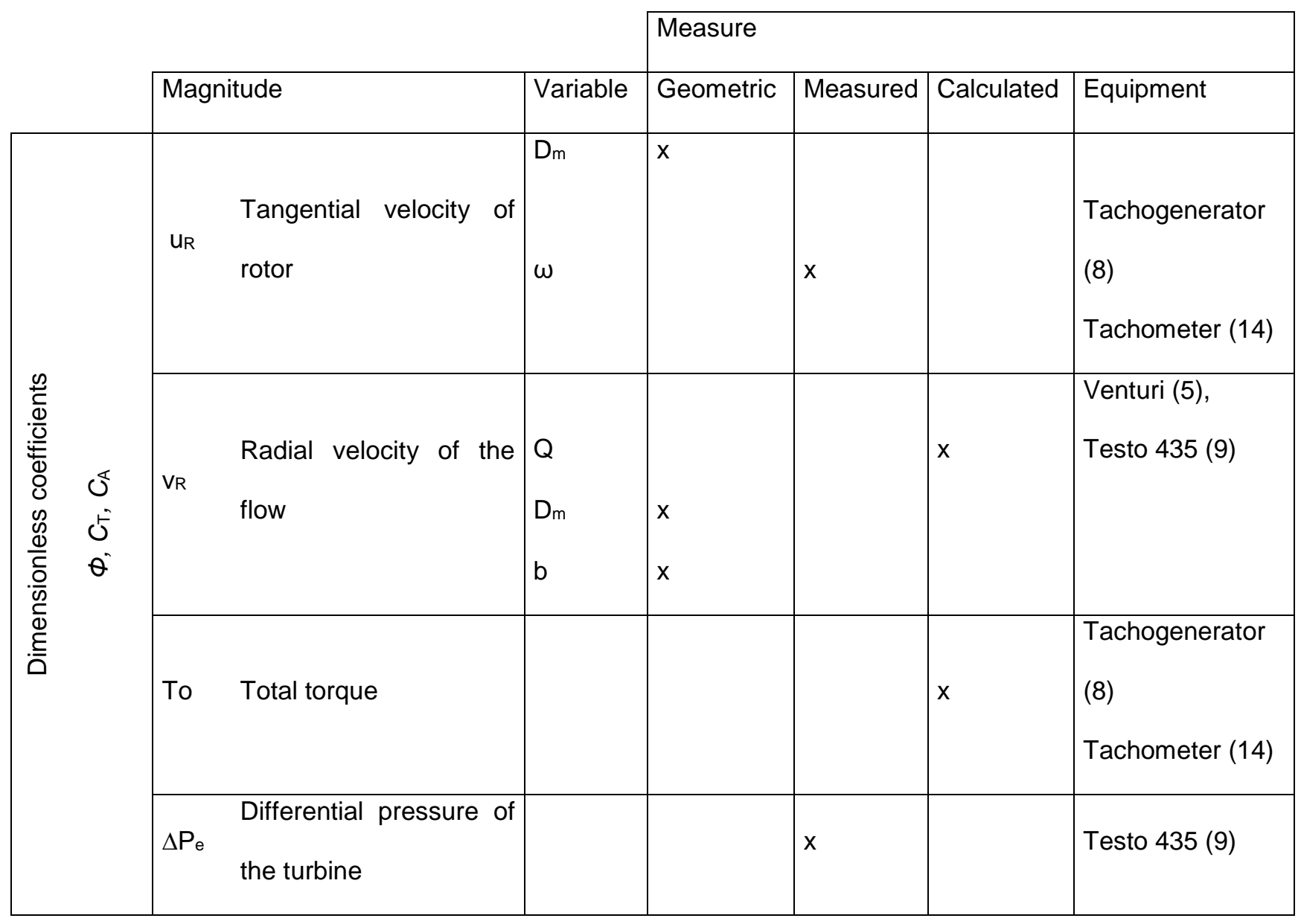




\begin{tabular}{|ll|l|l|l|l|l|}
$\theta_{\mathrm{a}} \quad$ Air temperature & & & $\mathrm{x}$ & & Testo 435 (9) \\
\hline $\mathrm{H}_{\mathrm{r}}$ & Relative humidity & & & $\mathrm{x}$ & & Testo 435 (9) \\
\hline $\mathrm{P}_{\mathrm{at}} \quad$ Atmospheric pressure & & & $\mathrm{x}$ & & Weather Station \\
\hline$\rho \quad$ Air density & $\theta_{\mathrm{a}}, \mathrm{H}_{\mathrm{r}}, \mathrm{P}_{\mathrm{at}}$ & & & $\mathrm{x}$ & \\
\hline
\end{tabular}

\subsection{Tachogenerator loss test}

This first test is performed to measure, for different rotating speeds, the voltage and current consumed by

the unloaded tachogenerator when running as a motor, for different rotating speeds. The voltage applied to the terminals of the tachogenerator is ranged between 0 and $85 \mathrm{~V} \mathrm{DC}$ and the resulting intensities are recorded to be processed according to the theory of electric machines, as follows:

$$
W_{A B}=V_{A B} \cdot I \quad W_{C U}=R_{i} \cdot I^{2} \quad W_{F E}+W_{M E C}=W_{A B}-W_{C U} \quad N=\frac{V_{A B}-R_{i} \cdot I}{V / \omega}
$$

Where $V_{A B}$ is the voltage applied in the terminals, $l$ is the intensity, $R_{i}$ is the internal resistance of the

tachogenerator, $W_{F E}$ is the iron loss, $W_{M E C}$ is the mechanical loss, $W_{C U}$ is the copper loss, $W_{A B}$ is the power in terminals, $N$ is the rotation speed and $V / \omega$ is the tachogenerator constant, equal to $0,06 \mathrm{~V} / \mathrm{rpm}$. Basic specifications of the Tachogenerator were extracted from the manufacturer documentation. The mechanical loss of the tachogenerator as a function of the rotating speed is represented with a solid line in Figure 11.

\subsection{Turbine+Tachogenerator loss test}

This second test is completed to determine the friction loss of the turbine. The turbine, decoupled from the test bench, is driven by the tachogenerator at different rotating speeds, recording the voltage and the intensity consumed. The difference between the input power and the calculated copper loss is the combined loss of the tachogenerator and the turbine.

Figure 11 also shows the results obtained as a function of the rotating speed (dashed line). As a consequence, the difference between the results obtained in the two former tests allow to obtain the mechanical loss of the turbine. 


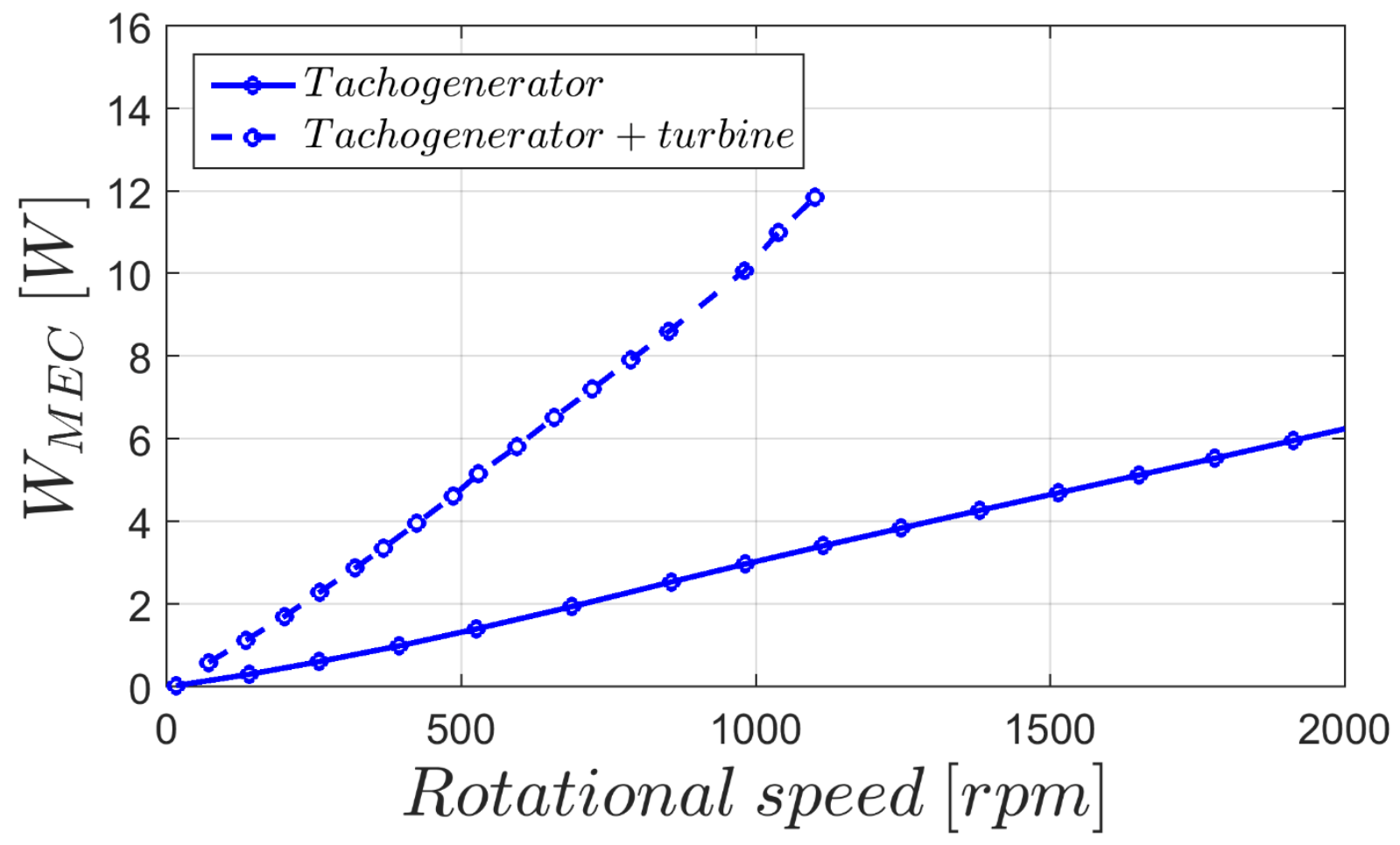

Figure 11.Turbine and tachogenerator losses

\subsection{Turbine test in direct mode}

313 In these tests, when the turbine is working in direct mode with a centrifugal flow (radially outwards), different 314 values of the flow rate coefficient are obtained changing the fan speed for the incoming flow, which makes 315 the turbine to rotate at velocities within the range of $122-1780 \mathrm{rpm}$.

316 Under steady conditions, the average values of differential pressure in the Venturi, the inlet static pressure 317 at the inlet and the rotating speed are measured and recorded to determine the input/output power. Note 318 that for this test, the mechanical loss is equal to the torque applied on the turbine blades, so the output 319 power can be extracted from the data obtained in the previous turbine+tachogenerator loss tests. Results for input power against the rotating speed are shown in Figure 12. 


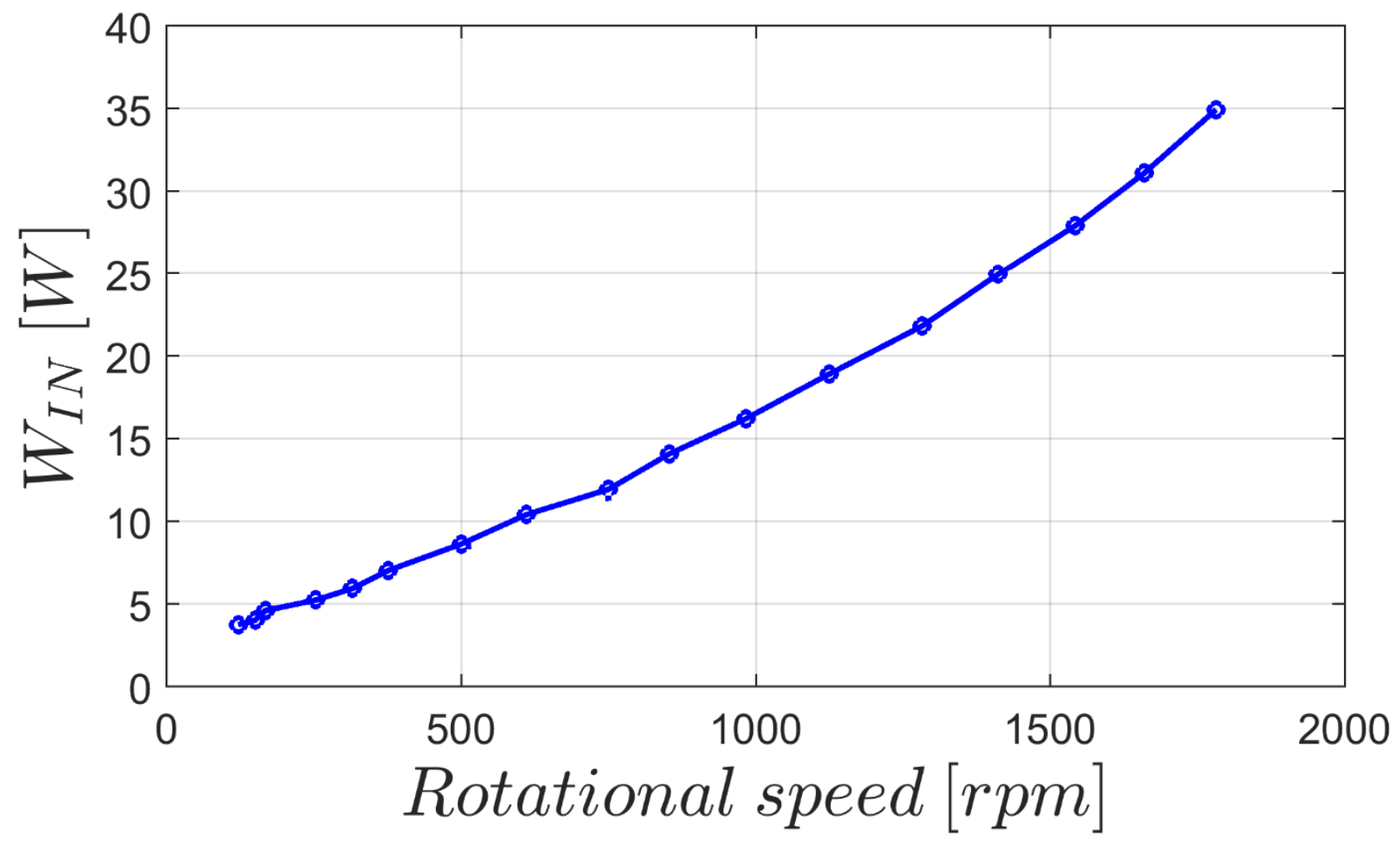

Figure 12. Power input in direct flow

324 Taking advantage of the previous tests to characterize the mechanical losses of the facility, the aerodynamic performance of the centrifugal turbine can be finally determined. The results have been obtained in a dimensionless form in Figure 13.

The range of flow coefficients tested, which spreads from 0.6 up to 2.5 , is consistent with the typical ranges of flow coefficients found for this type of OWC applications. Moreover, flow coefficients below 0.6 present a sharp drop in efficiency and with small magnitudes of both input and output power so they can be considered negligible for further analysis.

331 Note that the maximum total-to-static efficiency (right y-axis) reaches values over $50 \%$ (in the $\phi=0.7$ range), 332 exceeding by $10 \%$ the maximum efficiency reached by previous models (Rodríguez et al., 2018). This great 333 improvement is achieved due to the blade profile modifications which lead to a significant reduction in kinetic 334 energy loss at the outlet, as previously discussed. 


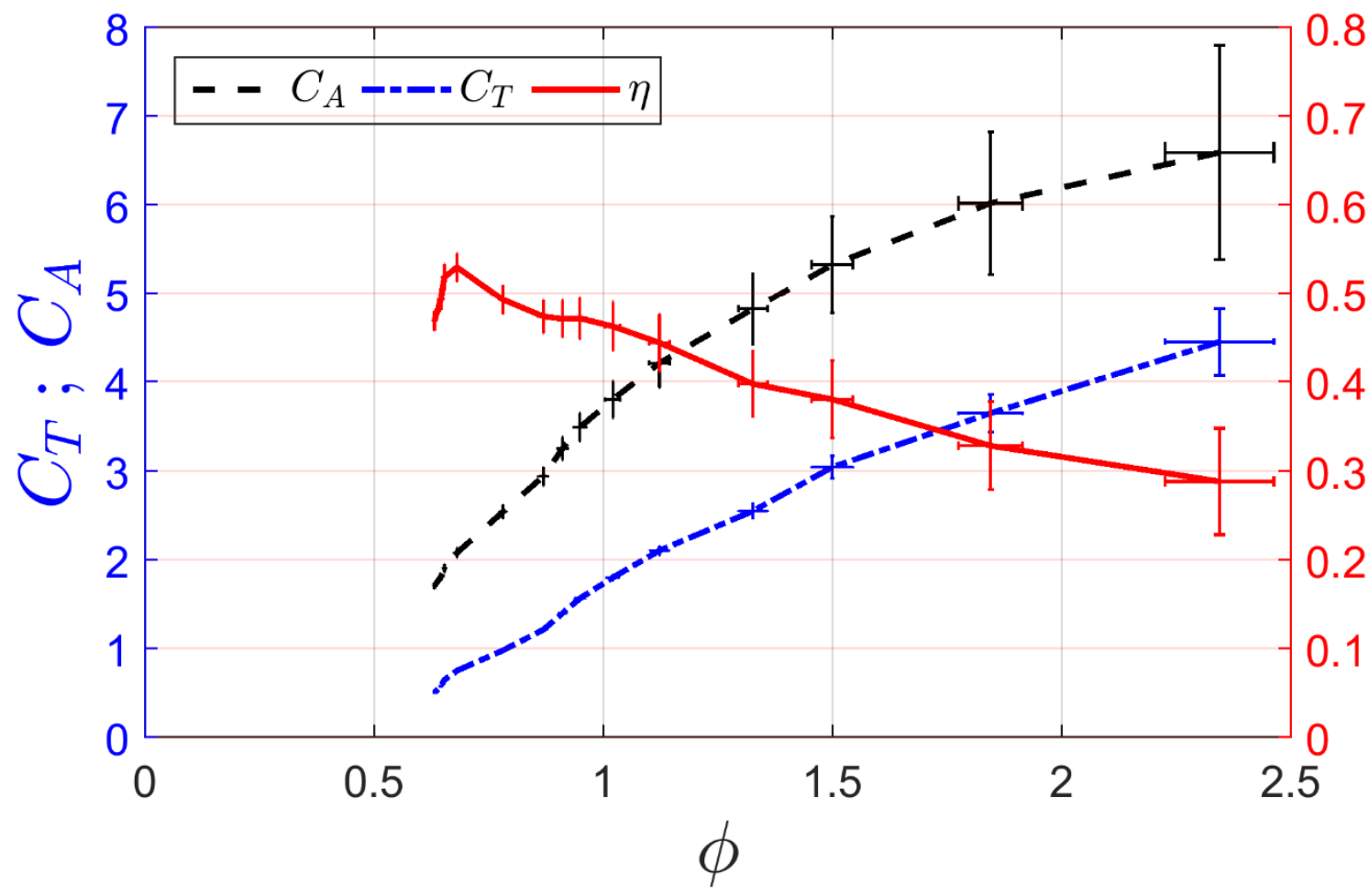

Figure 13. Measured coefficients in direct mode

339 Figure 13 also shows both torque and power coefficients with their corresponding uncertainties. In particular, the uncertainty of the flow coefficient is between $0.68 \%$ and $5 \%$, whereas the uncertainties of the torque coefficient and the input coefficient are between $0.88 \%-8.44 \%$ and $2.12 \%-18.32 \%$ respectively. The efficiency uncertainty is between $2.39 \%$ and $20.8 \%$.

\subsection{Turbine test in reverse mode}

This final test evaluates the performance of the turbine working in the reverse mode. To obtain a centrifugal flow, the duct is now connected to the aspirating region of the fan, while the turbine is driven by the tachogenerator that maintains the rotating speed between 420 and $547 \mathrm{rpm}$. In addition, the honeycomb is placed at the outlet of the turbine to reduce turbulence and suppress swirls motion, enhancing the Venturi measurement. For every flow rate tested, the following variables are recorded for further post-processing: static pressure at the outlet of the flow corrector, differential pressure in the Venturi and outlet voltage and current of the tachogenerator. After subtracting the mechanical loss, the performance curves of the turbine on reverse mode are obtained. 
The turbine pressure difference with respect the atmospheric condition is measured at the outlet of the flow honeycomb, discounting the honeycomb pressure losses previously assessed. Because the turbine works steadily at equilibrium (the rotating speed is constant), the available output power of the turbine can be measured from the power introduced in the tachogenerator, subtracting the losses by joule effect (eq. 9) and the tachogenerator + turbine mechanical friction (section 5.2). Finally, the torque is calculated by the expression:

$$
T_{O}=\frac{W_{r}}{\omega}
$$

Where $W_{r}$ is the brake power of the turbine and $\omega$ is the rotating speed.

The corresponding flow, torque and input coefficients are then calculated and shown in Figure 14 in dimensionless shape. Note that the torque coefficient is negative, which indicates that the turbine is braking. In this case, the efficiency is meaningless for the analysis so it is not represented here.

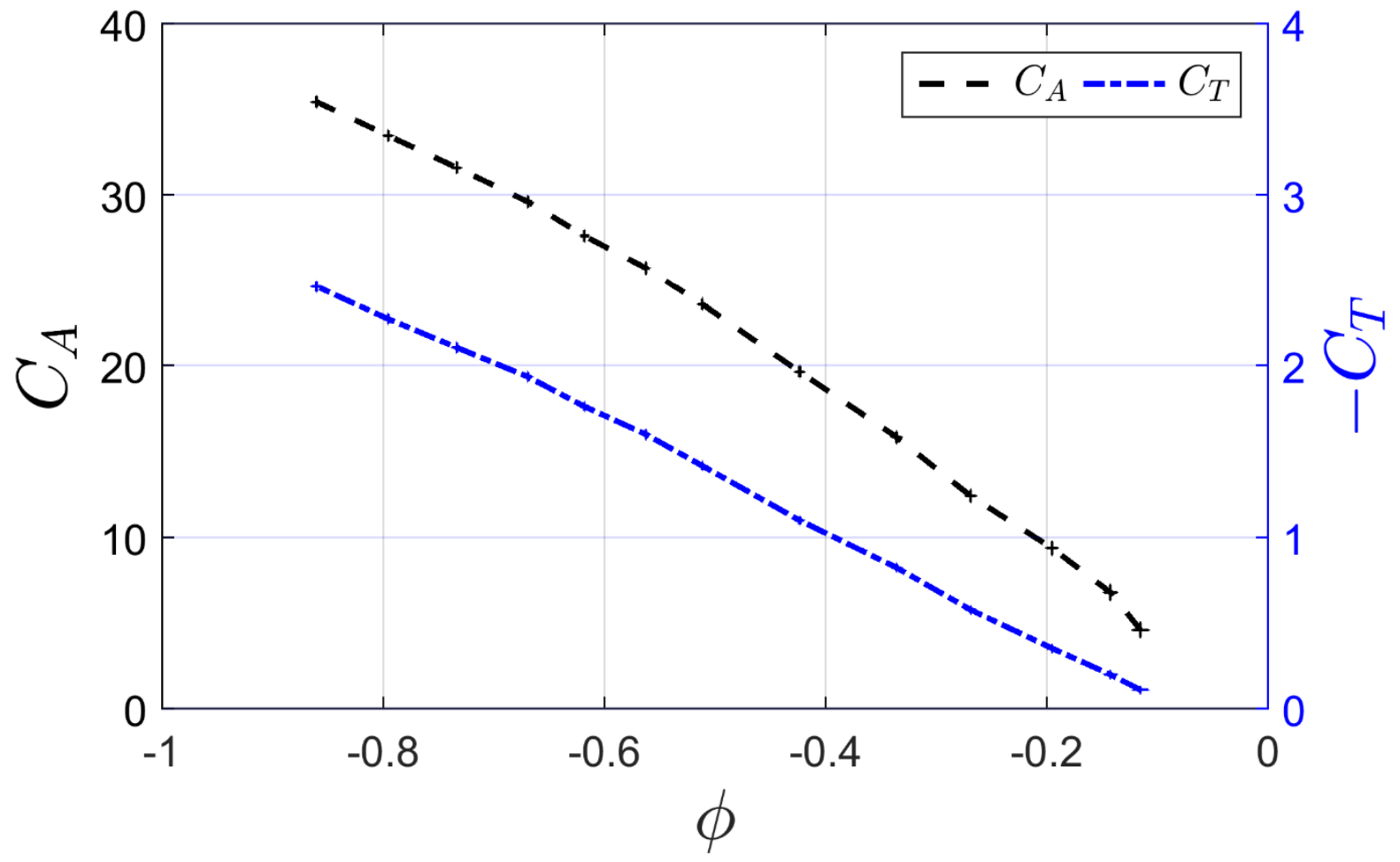

Figure 14. Torque and input coefficients in reverse mode

The figure also represents the evolution of the power coefficient, including the uncertainties for all the variables. In this case, the uncertainty of the flow coefficient is between $0,5 \%$ and $6 \%$. The uncertainty of the torque coefficient is between $1,3 \%$ and $16,4 \%$ while the uncertainty of the input coefficient is between 
$0,94 \%$ and $9,2 \%$. Note that in these tests, the smaller flow coefficients are achieved by reducing the flow rate, not by a large increase of the rotation speed such as in direct mode tests. This involves that the largest relative uncertainties correspond to the smaller values of the flow coefficient.

The comparison of Figure 13 and Figure 14 reveals the key point of this radial turbine to work in a twin turbine system. The input coefficient in the reverse mode is practically one order of magnitude higher than its value in the direct mode. This means that most of the flow rate generated by the OWC will flow through the turbine working in direct mode because the other turbine, working in reverse mode, will block the flow due to its large amount of loss.

\section{Study of the twin turbines system under non-steady conditions}

Following, the steady performance of the turbine is employed to characterize the overall, unsteady performance of the twin system according to the formulation presented in section 2 .

The evolution of the instantaneous volumetric efficiency and the direct, reverse and total flow rates in a dimensionless form is obtained over a wave cycle in Figure 15 for a given pressure coefficient in the OWC chamber $\left(\Phi_{T}=1.2\right)$.

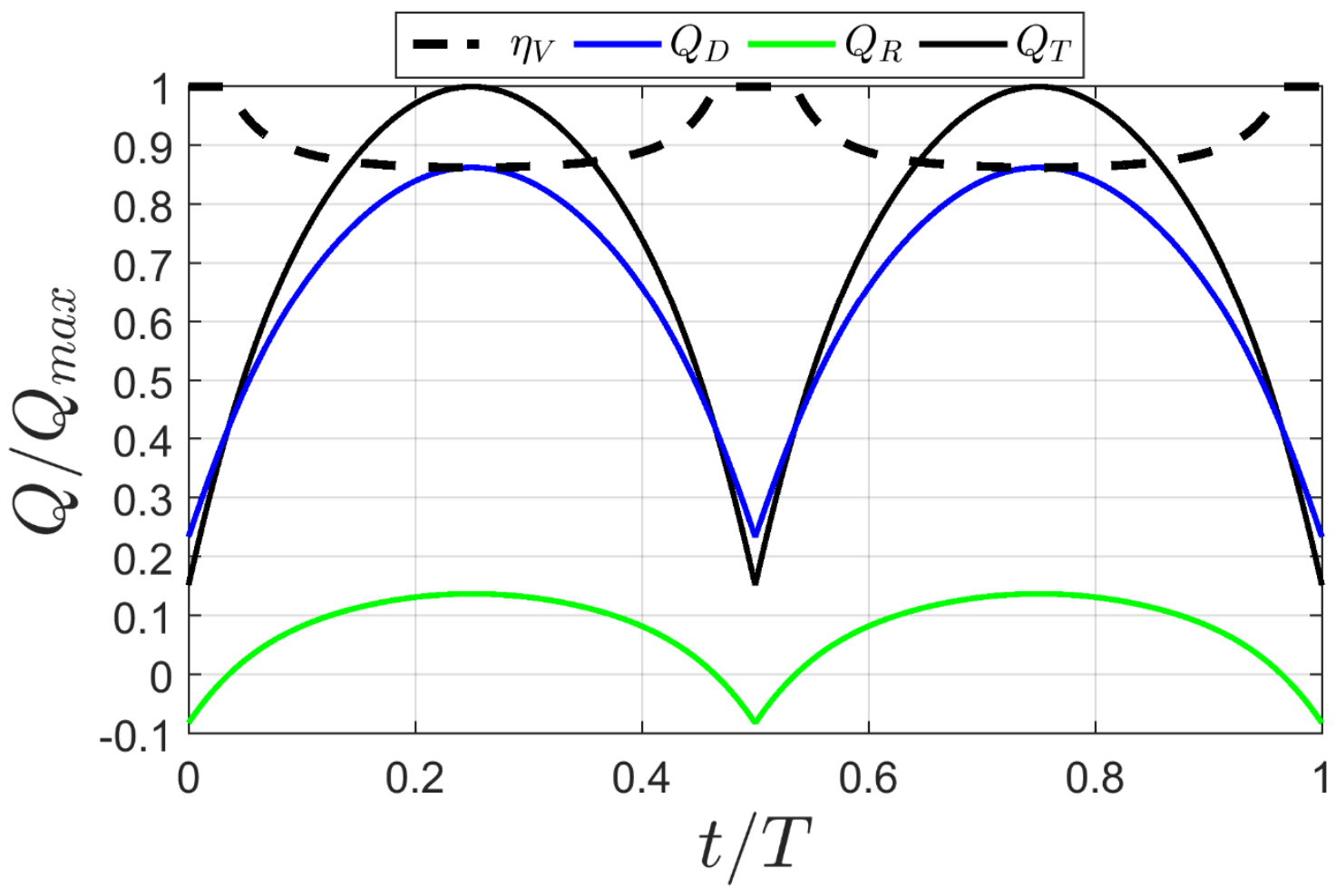


390 The maximum reverse flow rate is $13 \%$ of the total flow rate generated within the OWC. Therefore the $\eta_{V}$ 391 is, at least, above $87 \%$. This is a remarkable success when it is compared to the usual $60-65 \%$ reached by typical axial turbines (Takao et al., 2011); and also a significant progress with respect to the $76 \%$ of the previous radial model (Rodríguez et al., 2018).

Figure 16 shows the evolution of the efficiencies associated to the non-steady performance of the twin system, defined in eq. 6 , as a function of the flowrate coefficient for the system.

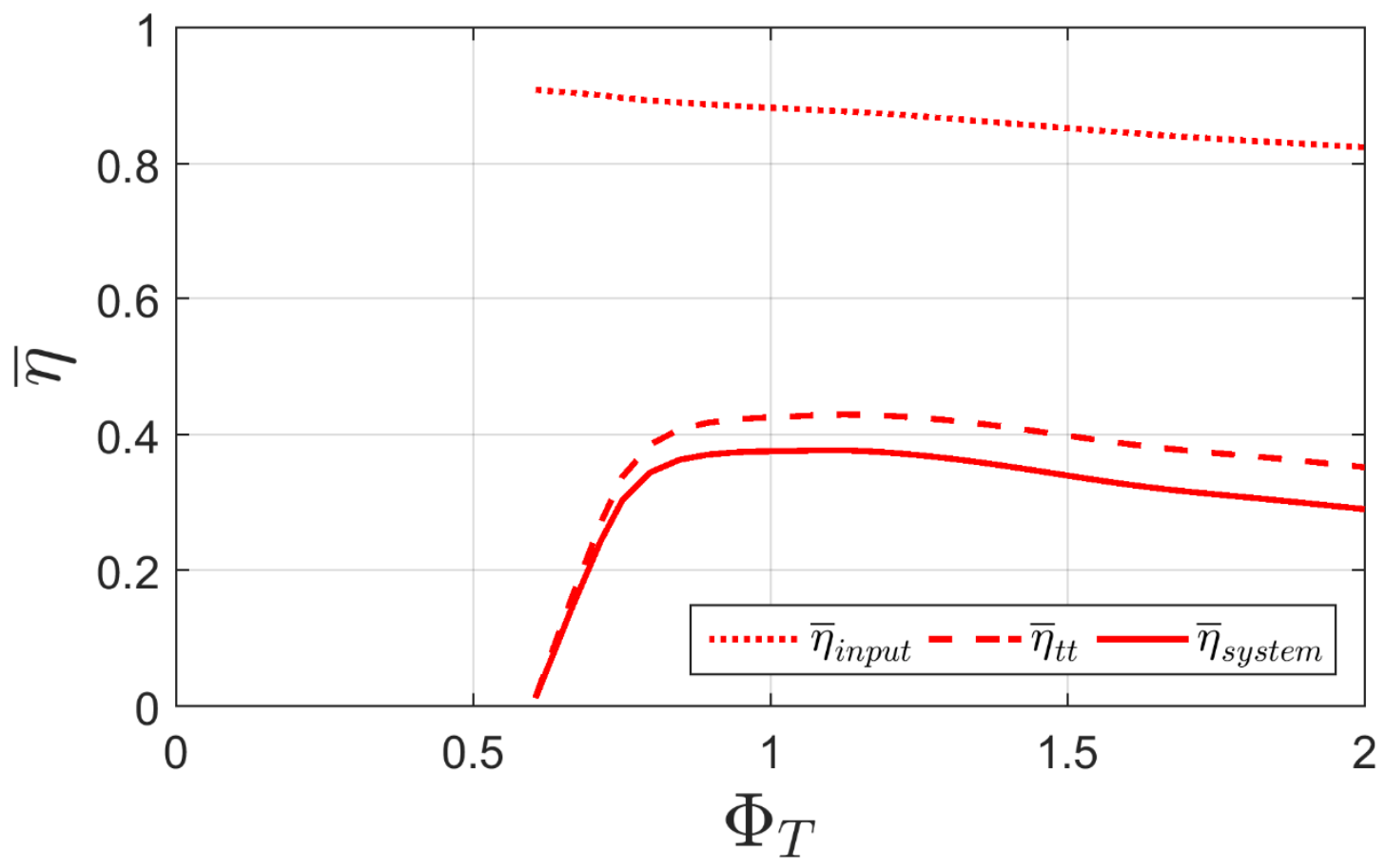

Figure 16. Efficiency of new twin radial system

The maximum non-steady efficiency reached by the twin turbines is 0.41 for a flow coefficient close to unity. Note that the system efficiency is practically reproducing the efficiency of the turbine due to the extremely high values of the volumetric efficiency. This fact represents the key contribution of this new design of a radial turbine for an OWC application. 
In this final section the results obtained for the present investigation are compared to those of the original 407 twin radial turbine (Rodríguez et al., 2018) and the twin axial turbine referred in (Takao et al., 2011).

\subsection{Steady conditions}

410 Firstly, the results of the new radial turbine are compared in Figure 17 with the quasi-steady performance 411 curve of the twin axial turbine from (Takao et al., 2011). There are four basic ideas to be highlighted from 412 the comparison of these steady results:

a) The new radial geometry produces a strong pressure difference in reverse mode which will involve a better performance working as a backflow preventer.

b) Although the torque in direct mode is being reduced from previous radial geometries, it is clear that the efficiency is better due to the reduction of loss. The maximum efficiency is up to $11 \%$ better than previous geometries (Rodríguez et al., 2018).

c) The torque in the reverse mode is neither suppressed nor reduced with respect to the previous radial turbine. However, the better performance as a backflow preventer will minimize this effect when working under non-steady conditions.

d) This new radial turbine is more efficient than any other radial turbine previously published for twin systems (at least, in a steady fashion). Moreover, the reached efficiency is almost as high as the typical values found for axial geometries (Takao et al., 2011). 

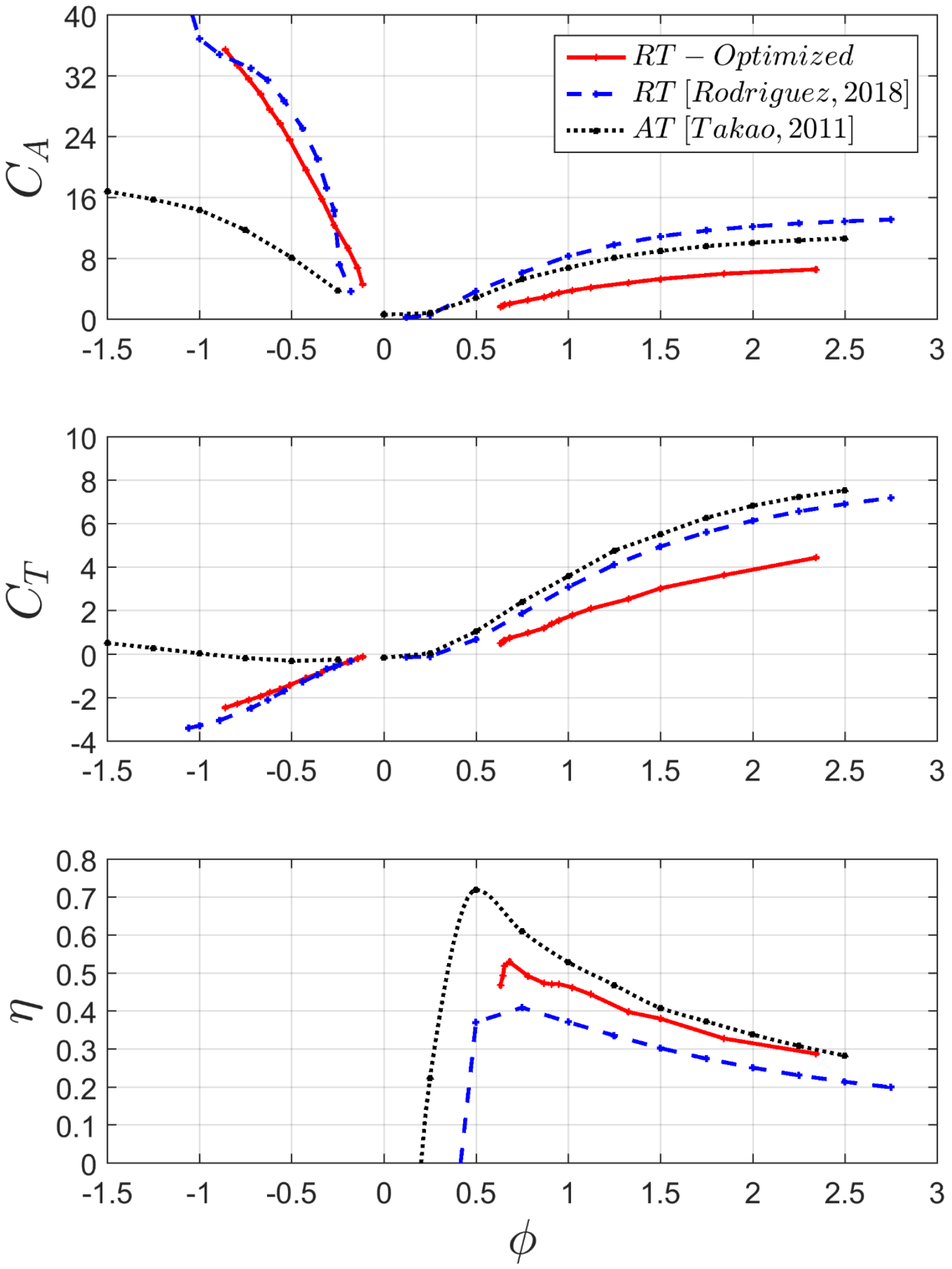

Figure 17. Compared results. Axial results from (Takao et al., 2011). 


\subsection{Non-steady conditions}

428 In this subsection, the non-steady performance of the twin system is evaluated as a function of the turbine 429 geometry to be installed. Basically, the results of the new radial turbine are shown with respect to the axial 430 turbine extracted from (Takao et al., 2011) and respect to the original radial turbine taken from (Rodríguez 431 et al., 2018). Other geometries have not been included in the graph for a better readability. The three 432 different efficiencies previously defined in eqs. 4-6 for non-steady conditions (i.e., the OWC input 433 efficiency $\bar{\eta}_{\text {input }}$, the net efficiency of each turbine $\bar{\eta}_{\text {turbine }}$, and the system efficiency $\bar{\eta}_{\text {system }}$ ) are 434 represented in Figure 18.

435 Focusing on the efficiency of the total system, $\bar{\eta}_{\text {system }}$, the comparison reveals that the results of the axial 436 turbine are improved by the new radial turbine presented in this work up to $2 \%$. This result could seem 437 surprisingly if only the efficiency of the turbine is taken into account because the efficiency of the axial 438 turbine is larger than the one reached by the radial one, as shown in the bottom plot of Figure 17. However, 439 as it was mentioned before, the system performance under non-steady conditions not only depends on the 440 turbine. The performance working as a backflow preventer, which is critical during the reverse mode, is 441 approximately between $18 \%$ an $30 \%$ better in case of the new radial turbine with respect to the axial one. 442 This implies that a larger part of the flow generated by the OWC is taken in advantage which finally turns 443 into a clear improvement of the system efficiency. 

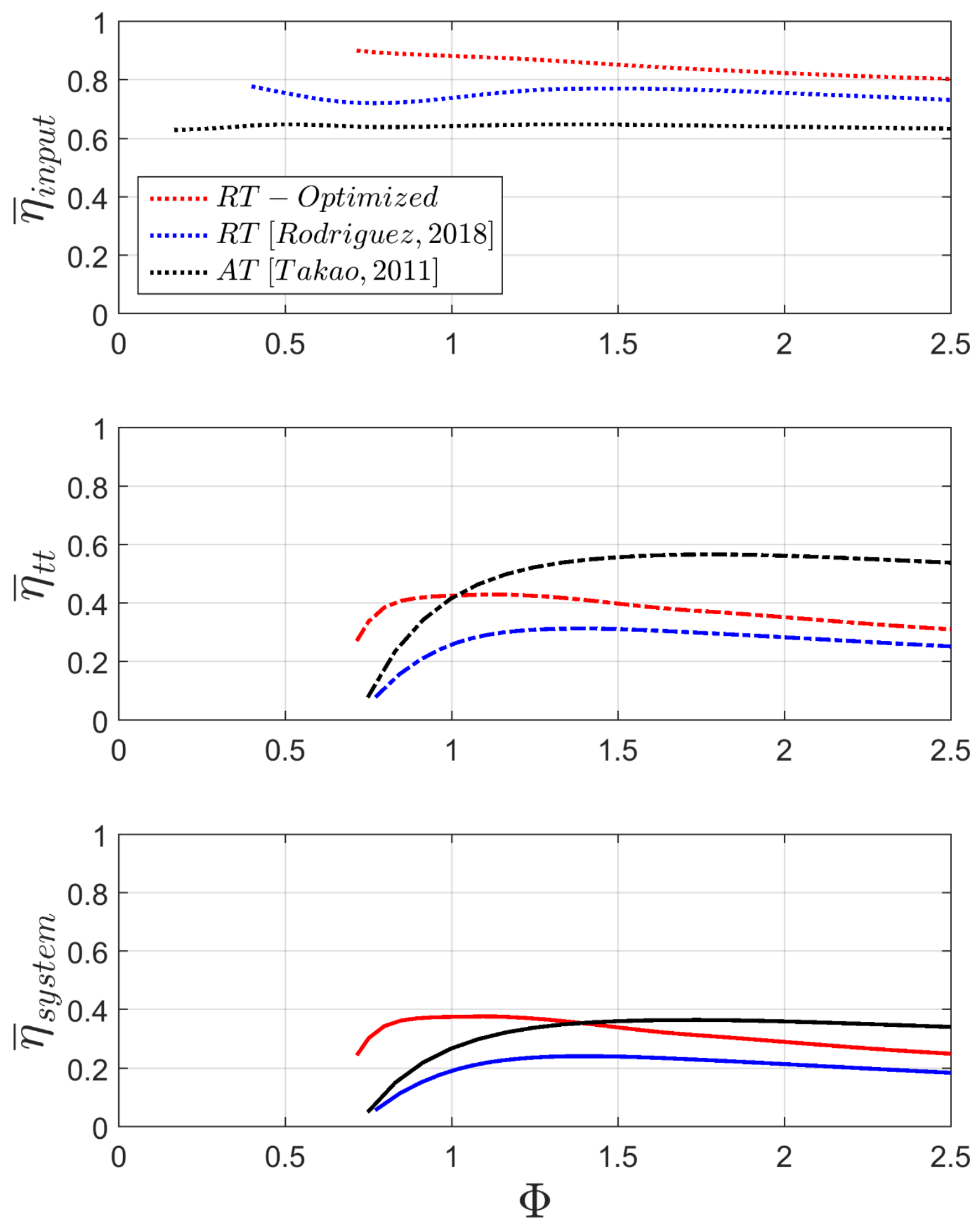


\section{Conclusions}

This paper presents the experimental study of an improved design for a radial turbine to be installed in a twin turbine system for an OWC device. The investigation concludes that radial impulse turbine can be a reliable and competitive solution for OWC applications.

452 The new radial turbine developed for this work includes some geometrical modifications intended to solve both mechanical and aerodynamic problems observed in a previous geometry. In particular, restrictions in 454 the flow circulation that led to significant penalties in the efficiency of the turbine have been now corrected. The experimental results presented confirm the expected improvements and the suitability of the centripetal sense to enhance the performance of the reverse mode. The flow blockage achieved with this configuration is extremely good and it reduces the importance of having negative torque while the air is flowing in centripetal direction because the flow rate is almost negligible in the reverse mode. Definitively, this is the key point that makes radial turbines particularly suitable for twin turbine systems.

460 Furthermore, the performance in direct mode has been also improved with a redesign of the rotor blades, exhibiting steady efficiencies (total-to-static) close to those obtained by typical axial turbines, supposedly much more efficient. The basic reason for this improvement in the direct mode has been the development of a new blade profile which improves the rotor efficiency and also reduces the kinetic energy loss at the 464 turbine outlet.

All these improvements had led to a clear progress in the performance of twin turbine systems under nonsteady conditions, reaching up to a $38 \%$ of total system efficiency, which is even higher than previous results obtained by other axial turbines available in the literature. Therefore, radial turbines have demonstrated a definitive potential to be a real solution for twin turbine systems in OWC devices.

\section{Acknowledgments}

The authors acknowledge the support provided by "Centro Integrado de F.P. Mantenimiento y Servicios a

472 la Producción de Langreo", with special mention to its Director, Mr. F. Fanjul.

\section{References:}

475 Drew, B., Plummer, A.R., Sahinkaya, M.N., 2009. A review of wave energy converter technology. Proc. Inst. Mech. Eng. Part A J. Power Energy 223, 887-902. doi:10.1243/09576509JPE782

477 Falcão, A.F. d. O., 2010. Wave energy utilization: A review of the technologies. Renew. Sustain. Energy 
Rev. 14, 899-918. doi:10.1016/j.rser.2009.11.003

Falcão, A.F.O., Gato, L.M.C., Henriques, J.C.C., Borges, J.E., Pereiras, B., Castro, F., 2015. A novel twinrotor radial-inflow air turbine for oscillating-water-column wave energy converters. Energy 93, 21162125. doi:10.1016/j.energy.2015.10.046

Falcão, A.F.O., Henriques, J.C.C., 2016. Oscillating-water-column wave energy converters and air turbines: A review. Renew. Energy 85, 1391-1424. doi:10.1016/j.renene.2015.07.086

Iglesias, G., Carballo, R., 2014. Wave farm impact: The role of farm-to-coast distance. Renew. Energy 69, 375-385. doi:10.1016/j.renene.2014.03.059

IPCC, 2012. Renewable energy sources and climate change mitigation: special report of the Intergovernmental Panel on Climate Change. Choice Rev. Online 49, 49-6309-49-6309. doi:10.5860/CHOICE.49-6309

Jayashankar, V., Anand, S., Geetha, T., Santhakumar, S., Jagadeesh Kumar, V., Ravindran, M., Setoguchi, T., Takao, M., Toyota, K., Nagata, S., 2009. A twin unidirectional impulse turbine topology for OWC based wave energy plants. Renew. Energy 34, 692-698. doi:10.1016/j.renene.2008.05.028

López, I., Andreu, J., Ceballos, S., Martínez De Alegría, I., Kortabarria, I., 2013. Review of wave energy technologies and the necessary power-equipment. Renew. Sustain. Energy Rev. 27, 413-434. doi:10.1016/j.rser.2013.07.009

Mala, K., Jayaraj, J., Jayashankar, V., Muruganandam, T.M., Santhakumar, S., Ravindran, M., Takao, M., Setoguchi, T., Toyota, K., Nagata, S., 2011. A twin unidirectional impulse turbine topology for OWC based wave energy plants - Experimental validation and scaling. Renew. Energy 36, 307-314. doi:10.1016/j.renene.2010.06.043

Marigorta, E.B., Tajadura, R.B., 1994. Análisis de incertidumbre en mecánica de fluidos. Universidad de Oviedo, Gijón.

Palha, A., Mendes, L., Fortes, C.J., Brito-Melo, A., Sarmento, A., 2010. The impact of wave energy farms in the shoreline wave climate: Portuguese pilot zone case study using Pelamis energy wave devices. Renew. Energy 35, 62-77. doi:10.1016/j.renene.2009.05.025

Pereiras, B., Castro, F., el Marjani, A., Rodríguez, M.A., 2011. Tip Clearance Effect on the Flow Pattern of a Radial Impulse Turbine for Wave Energy Conversion. J. Turbomach. 133, 41019. doi:10.1115/1.4002409

Pereiras, B., Valdez, P., Castro, F., 2014. Numerical analysis of a unidirectional axial turbine for twin turbine 
509 Raghunathan, S., 1995. The wells air turbine for wave energy conversion. Prog. Aerosp. Sci. 31, 335-386. doi:10.1016/0376-0421(95)00001-F

511 Rodríguez, L., Pereiras, B., Fernández-Oro, J., Castro, F., 2018. Viability of unidirectional radial turbines 512 for twin-turbine configuration of OWC wave energy converters. Ocean Eng. 154, 288-297. 513 doi:10.1016/j.oceaneng.2018.02.010

514 Setoguchi, T., Takao, M., 2006. Current status of self rectifying air turbines for wave energy conversion. $515 \quad$ Energy Convers. Manag. 47, 2382-2396. doi:10.1016/j.enconman.2005.11.013

516 Takao, M., Setoguchi, T., 2012. Air Turbines for Wave Energy Conversion. Int. J. Rotating Mach. 2012, 1$517 \quad$ 10. doi: $10.1155 / 2012 / 717398$

518 Takao, M., Takami, A., Okuhara, S., Setoguchi, T., 2011. A twin unidirectional impulse turbine for wave 519 energy conversion. J. Therm. Sci. 20, 394-397. doi:10.1007/s11630-011-0486-1

520 Wells, A.A., 1980. Rotary transducers. Patent no US4221538 A. 
527 This document is a pre-print version of the scientific paper published by Elsevier. It has been released by the authors to fulfill all the publisher requirements established for Article Sharing:

https://www.elsevier.com/about/policies/sharing

\section{(c) (†) $\ominus$}

(C) 2019. This manuscript version is made available under the Creative Commons Attribution-NonCommercial-NoDerivatives 4.0 International License (CC-BY-NC-ND 4.0 license) 